\title{
COMBINATORIAL AND GROUP-THEORETIC COMPACTIFICATIONS OF BUILDINGS
}

\author{
PIERRE-EMMANUEL CAPRACE* AND JEAN LÉCUREUX
}

\begin{abstract}
Let $X$ be a building of arbitrary type. A compactification $\mathscr{C}_{\text {sph }}(X)$ of the set $\operatorname{Res}_{\mathrm{sph}}(X)$ of spherical residues of $X$ is introduced. We prove that it coincides with the horofunction compactification of $\operatorname{Res}_{\mathrm{sph}}(X)$ endowed with a natural combinatorial distance which we call the root-distance. Points of $\mathscr{C}_{\mathrm{sph}}(X)$ admit amenable stabilisers in $\operatorname{Aut}(X)$ and conversely, any amenable subgroup virtually fixes a point in $\mathscr{C}_{\mathrm{sph}}(X)$. In addition, it is shown that, provided $\operatorname{Aut}(X)$ is transitive enough, this compactification also coincides with the group-theoretic compactification constructed using the Chabauty topology on closed subgroups of Aut $(X)$. This generalises to arbitrary buildings results established by Y. Guivarc'h and B. Rémy [GR06] in the Bruhat-Tits case.
\end{abstract}

\section{CONTENTS}

Introduction

Acknowledgment

1. The root-distance on spherical residues

1.1. Preliminaries

1.2. The root-distance

2. Combinatorial compactifications 7

2.1. Definition 7

2.2. Projecting from infinity 11

2.3. Extending the notion of sectors to arbitrary buildings 12

2.4. Sectors and half-apartments 15

2.5. Incidence properties of sectors 19

2.6. Covering the combinatorial compactifications with apartments 19

3. Horofunction compactifications 20

4. Group-theoretic compactifications 22

4.1. The Chabauty topology 22

4.2. Locally finite groups 22

4.3. Stabilisers of points at infinity 23

4.4. Description of the group-theoretic compactification 25

5. Comparison to the refined visual boundary 28

5.1. Constructing buildings in horospheres 28

5.2. A stratification of the combinatorial compactifications 29

5.3. Comparison to the refined visual boundary 30

6. Amenability of stabilisers 32

1991 Mathematics Subject Classification. 20E42; 20G25, 22E20, 22F50, 51E24.

Key words and phrases. Compactification, building, Chabauty topology, amenable group.

*F.N.R.S. Research Associate. 
Appendix A. Combinatorial compactifications of CAT(0) cube complexes

\section{INTRODUCTION}

The best known and probably most intuitively obvious compactification of a non-compact Riemannian symmetric space $M$ is the visual compactification $\bar{M}=M \cup \partial_{\infty} M$, whose points at infinity consist in equivalence classes of geodesic rays at finite Hausdorff distance of one another. Following Gromov [BGS85], this compactification may be identified with the horofunction compactification $\mathscr{C}_{\text {horo }}(M)$, whose points at infinity are Busemann functions. This canonical identification holds in fact for any CAT(0) metric space, see [BH99, Theorem II.8.13].

Another way to approach to visual compactification of $M$ is the following. Using the visual map which associates to every pair of points $p, q \in M$ the direction at $p$ of the geodesic segment $[p, q]$, it is possible to associate to every point of $M$ a unique element of the unit tangent ball bundle over $M$. The total space of this bundle being compact, one obtains a compactification by passing to the closure of the image of $M$; this coincides with the visual compactification $\bar{M}$. Here again, the construction has a natural analogue which makes sense in any locally compact CAT(0) space $X$ provided that the space of directions $\Sigma_{p} X$ at every point $p \in X$ is compact. This condition is automatically satisfied if $X$ is geodesically complete (i.e. every geodesic segment may be extended to a bi-infinite geodesic line, which need not be unique) or if $X$ has the structure of a CAT(0) cell complex. In the latter case, each space of direction $\Sigma_{p} X$ is endowed with the structure of a finite cell complex.

This suggests to modify the above construction of the visual compactification as follows. Assume $X$ is a locally finite $\mathrm{CAT}(0)$ cell complex. Then the space of direction $\Sigma_{p} X$ has a cellular structure; one denotes by $\operatorname{St}(p)$ the corresponding set of cells. Associating to each point its support, one obtains a canonical map $\Sigma_{p} X \rightarrow \operatorname{St}(p)$. Pre-composing with the aforementioned visual map, one obtains a map $X \rightarrow \prod_{p \in X} \operatorname{St}(p)$. The closure of this map is called the combinatorial compactification of $X$. It should be noted that the above map is not injective in general: two points with the same support are identified.

The main purpose of this paper is to pursue this line of thoughts in the special case of buildings of arbitrary type. Similar developments in the case of CAT(0) cube complexes are carried out in the Appendix.

In the case of buildings, the relevant simplices are the so-called residues of spherical type, also called spherical residues for short. The above combinatorial compactification thus yields a compactification of the set $\operatorname{Res}_{\mathrm{sph}}(X)$ of all spherical residues, and the above 'visual map' $\operatorname{Res}_{\mathrm{sph}}(X) \rightarrow \prod_{\sigma \in \operatorname{Res}_{\mathrm{sph}}(X)} \operatorname{St}(\sigma)$ may be canonically defined in terms of the combinatorial projection. Its closure is the the combinatorial compactification and will be denoted by $\mathscr{C}_{\mathrm{sph}}(X)$.

The set $\operatorname{Res}_{\mathrm{sph}}(X)$ may moreover be endowed in the canonical with the structure of a discrete metric space. For example, a graph structure on $\operatorname{Res}_{\mathrm{sph}}(X)$ is obtained by declaring two residues adjacent if one is contained in the other. We shall introduce a sligthly different 
distance, called the root-distance which has the advantage that its restriction to the chamberset $\mathrm{Ch}(X)$ coincides with the gallery distance (see Section 1). As any proper metric space, the discrete metric space $\operatorname{Res}_{\mathrm{sph}}(X)$ admits a horofunction compactification This turns out to coincide with the combinatorial compactification (see Theorem 3.1).

It is important to remark that the combinatorial compactification does not coincide with the visual one. Although there are elementary ways to establish the latter fact, strong evidence is provided by the following result (see Theorem 6.1).

Theorem A. Let $X$ be a locally finite building. Then every amenable subgroup of $\operatorname{Aut}(X)$ has a finite index subgroup which fixes some point in $\mathscr{C}_{\mathrm{sph}}(X)$.

Conversely, the full stabiliser of every point of $\mathscr{C}_{\mathrm{sph}}(X)$ is a closed amenable subgroup.

In the special case of Bruhat-Tits buildings, a similar statement was established in [GR06, Theorem 33] using another compactification called the group-theoretic compactification. The construction of the latter goes back to an idea of Y. Guivarc'h in the case of symmetric spaces and be be outlined as follows. A symmetric space $M$ embeds in the space of closed subgroups of $\operatorname{Isom}(M)$ by attaching to each point its isotropy group. Since the space of closed subgroups endowed with Chabauty topology is compact, one obtains a compactification by passing to the closure. This yields the group-theoretic compactification $\mathscr{C}_{\mathrm{gp}}(M)$. This turns out to be equivariantly isomorphic to the maximal Satake and Furstenberg compactifications (see [GJT98], [BJ06]). In the case of buildings, since points with the same support have identical stabilisers, this approach cannot offer better than a compactification of the set $\operatorname{Res}_{\mathrm{sph}}(X)$.

Theorem B. Assume that $\operatorname{Aut}(X)$ acts strongly transitively. The group-theoretic compactification $\mathscr{C}_{\mathrm{gp}}(X)$ is $\operatorname{Aut}(X)$-equivariantly homeomorphic to the maximal combinatorial compactification $\mathscr{C}_{\mathrm{sph}}(X)$. More precisely, a sequence $\left(R_{n}\right)$ of spherical residues converges to some $\xi \in \mathscr{C}_{\mathrm{sph}}(X)$ if and only if the sequence of their stabilisers $\left(G_{R_{n}}\right)$ converges to the locally finite radical of $G_{\xi}$ in the Chabauty topology.

Recall that the locally finite radical of a locally compact group $G$ is the unique subgroup $\operatorname{Rad}_{\mathrm{LF}}(G)$ which is (topologically) locally finite (i.e. all of whose finitely generated subgroups are relatively compact), normal and maximal for these properties. It was shown in [Cap07] that a closed subgroup $H$ of $\operatorname{Aut}(X)$ is amenable if and only if $H / \operatorname{Rad}_{\mathrm{LF}}(H)$ is virtually Abelian.

It is shown in [GR06] that the group-theoretic compactifications may be canonically identified with the polyhedral compactification constructed by E. Landvogt in [Lan96]. Theorem B may be viewed as an extension of this to the case of arbitrary buildings.

A central tool introduced in this work to study the combinatorial compactification is the notion of combinatorial sectors, which extend to the general case the classical notion of sectors in Bruhat-Tits theory. Given a point $\xi \in \mathscr{C}_{\mathrm{sph}}(X)$, we associate to every $x \in \operatorname{Res}_{\mathrm{sph}}(X)$ as sector $Q(x, \xi)$ based at $x$ and pointing to $\xi$ (see Section 2.6). Every sector is contained in an apartment; the key property is that the collection of all sectors pointing to $\xi \in \mathscr{C}_{\text {sph }}(X)$ is filtering; in other words any two sectors pointing to $\xi$ contain a common subsector (see Proposition 2.30).

We emphasize that all of our considerations are valid for arbitrary buildings and are of elementary nature; in particular, no use is made of the theory of algebraic groups. Moreover, as it will appear in the core of the paper, most of the results remain valid for buildings which are not necessarily locally finite (in that case, one uses the term bordification instead of compactification). 
The paper is organised as follows. In a first section we introduce and study the properties of a combinatorial distance on $\operatorname{Res}_{\mathrm{sph}}(X)$ which we call the root-distance. The next section is devoted to the combinatorial compactification. Combinatorial sectors are introduced and used to prove that every point of $\mathscr{C}_{\mathrm{sph}}(X)$ may be attained as the limit of some sequence of residues all contained in a common apartment. The third section is devoted to the horofunction compactification and proves that in the case of $\operatorname{Res}_{\mathrm{sph}}(X)$ the combinatorial and horofunction compactifications coincide. Chabauty topology is studied in the next section, whose main goal is to prove Theorem B. The next section studies the relationship between the visual boundary and the combinatorial compactification. The main results are a stratification of the combinatorial compactification (Theorem 5.5) and a description of $\mathscr{C}_{\mathrm{sph}}(X)$ as the quotient of the refined visual boundary of $X$ which is a refined version of the visual boundary introduced in [Cap07] for arbitrary CAT(0) spaces. These results are used in the final section which proves Theorem A. Finally, the Appendix outlines similar results in the case of finite-dimensional CAT(0) cube complexes.

Acknowledgment. The second author is very grateful to Bertrand Rémy for his constant support.

\section{THE ROOT-Distance ON SPHERICAL RESIDUES}

1.1. Preliminaries. Throughout this paper we let $X$ be an arbitrary building of finite rank and $G$ be its full automorphism group. We denote by $\operatorname{Ch}(X)\left(\operatorname{resp} . \operatorname{Res}_{1}(X), \operatorname{Res}_{\mathrm{sph}}(X)\right)$ the set of chambers (resp. panels, spherical residues) of $X$. Given a residue $\sigma$ of $X$, the star of $\sigma$, denoted by $\operatorname{St}(\sigma)$, is the set of all residues containing $\sigma$ in their boundaries, see [Tit74, §1.1]. We recall that, in the chamber system approach to buildings, which is dual to the simplicial approach, a residue is viewed as a set of chambers and the star is then nothing but the set of all residues contained in $\sigma$. This has no influence on the subsequent considerations and the reader should feel free to adopt the point of view which he/she is most comfortable with.

1.2. The root-distance. Our first task is to introduce a combinatorial distance on the set $\operatorname{Res}_{\mathrm{sph}}(X)$ of spherical residues. A natural way to obtain such a distance is by considering the incidence graph of spherical residues, namely the graph with vertex set $\operatorname{Res}_{\mathrm{sph}}(X)$ where two residues are declared to be adjacent if one is contained in the other. However, the disadvantage of this graph is that the natural embedding of $\mathrm{Ch}(X)$ in $\operatorname{Res}_{\mathrm{sph}}(X)$ is not isometric, when $\operatorname{Ch}(X)$ is endowed with the gallery distance. This causes some technical difficulties which we shall avoid by introducing an alternative distance on $\operatorname{Res}_{\mathrm{sph}}(X)$.

Given $R_{1}, R_{2} \in \operatorname{Res}_{\mathrm{sph}}(X)$, let $A$ be an apartment containing them both. We denote by $\Phi_{A}\left(R_{1}, R_{2}\right)$ the set of all half-apartments of $A$ containing $R_{1}$ but not $R_{2}$. This set is empty if and only if $R_{1}$ is contained in $R_{2}$, since every residue coincides with the intersection of all half-apartments containing it. Notice moreover that the cardinality of the sets $\Phi_{A}\left(R_{1}, R_{2}\right)$ and $\Phi_{A}\left(R_{2}, R_{1}\right)$ is independent of the choice of $A$. We define the root-distance $d\left(R_{1}, R_{2}\right)$ between $R_{1}$ and $R_{2}$ to be half of the sum of their cardinalities. In symbols:

$$
d\left(R_{1}, R_{2}\right)=\frac{1}{2}\left|\Phi_{A}\left(R_{1}, R_{2}\right)\right|+\frac{1}{2}\left|\Phi_{A}\left(R_{2}, R_{1}\right)\right| .
$$

Clearly the restriction of the root-distance to the chamber set coincides with the gallery distance. However, checking that the root-distance indeed defines a metric on $\operatorname{Res}_{\mathrm{sph}}(X)$ requires some argument (see Proposition 1.2). Before collecting this together with some other basic facts on the root distance, we introduce some additional terminology. 
A set of spherical residues $\mathcal{R} \subset \operatorname{Res}_{\mathrm{sph}}(X)$ is called closed if for all $R_{1}, R_{2} \in \operatorname{Res}_{\mathrm{sph}}(X)$, we have

$$
R_{1} \subset R_{2} \in \mathcal{R} \Rightarrow R_{1} \in \mathcal{R}
$$

It is called convex if it is closed and if for all $R_{1}, R_{2} \in \mathcal{R}$, we have $\operatorname{proj}_{R_{1}}\left(R_{2}\right) \in \mathcal{R}$, where proj denotes the combinatorial projection (see [Tit74, §3.19]) or [AB08, Section 4.9]). We recall that by definition we have

$$
\operatorname{proj}_{R_{1}}\left(R_{2}\right)=\bigcap\left\{\operatorname{proj}_{R_{1}}(C) \mid C \in \mathrm{Ch}(X) \cap \operatorname{St}\left(R_{2}\right)\right\},
$$

which allows one to recover the combinatorial projections amongst arbitrary residues from projections of chambers.

Since any intersection of closed (resp. convex) subsets is closed (resp. convex) and since the whole set $\operatorname{Res}_{\mathrm{sph}}(X)$ is so, it makes sense to consider the closure (resp. the convex hull) of a subset $\mathcal{R} \subset \operatorname{Res}_{\mathrm{sph}}(X)$, which we denote by $\overline{\mathcal{R}}(\operatorname{resp}$. $\operatorname{Conv}(\mathcal{R})$ ). The convex hull of two residues $R_{1}, R_{2}$ is denoted by $\operatorname{Conv}\left(R_{1}, R_{2}\right)$. Given an apartment $A$ containing $R_{1} \cup R_{2}$, the convex hull Conv $\left(R_{1}, R_{2}\right)$ coincides with the intersection of all half-apartments of $A$ containing $R_{1} \cup R_{2}$. The following basic fact provides a convenient characterisation of the combinatorial projection:

Lemma 1.1. Given two (spherical) residues $R, T$, the combinatorial projection $\operatorname{proj}_{R}(T)$ coincides with the unique maximal residue containing $R$ and contained in $\operatorname{Conv}(R, T)$.

'Maximal' should be understood as 'of maximal possible dimension', i.e. of minimal possible rank.

Proof. Let $A$ be an apartment containing $\operatorname{Conv}(R, T)$ and $\sigma_{1}, \sigma_{2} \in \operatorname{Conv}(R, T)$ be two maximal residues containing $R$. Assume $\sigma_{1}$ and $\sigma_{2}$ are distinct. Then there is a half-apartment $\alpha$ of $A$ containing one but not the other. Without loss of generality $\sigma_{1} \subset \alpha$ but $\sigma_{2} \not \subset \alpha$. Since $R \subset \sigma_{1} \cap \sigma_{2}$ we have $R \subset \partial \alpha$. Therefore, if $T \subset \alpha$, then $\operatorname{Conv}(R, T) \subset \alpha$ which contradicts $\sigma_{2} \not \subset \alpha$. Thus $T$ meets in the interior of $-\alpha$. In particular, so does $\operatorname{proj}_{\sigma_{1}}(T)$. Since the latter is a spherical residue containing $\sigma_{1} \supset R$, we have $\sigma_{1}=\operatorname{proj}_{\sigma_{1}}(T)$, which contradicts the fact that $\sigma_{1}$ is contained in $\alpha$.

This confirms that there is a unique maximal residue $\sigma \in \operatorname{Conv}(R, T)$ containing $R$. Since $\operatorname{proj}_{R}(T) \supset R$, we have thus $\operatorname{proj}_{R}(T) \subset \sigma$. If the latter inclusion were proper, then there would exist some root $\beta$ containing $\operatorname{proj}_{R}(T)$ but not $\sigma$. In particular $R$ and $\operatorname{proj}_{R}(T)$ are contained in the wall $\partial \beta$. This implies that $T$ is also contained in $\partial \beta$. Therefore so is $\operatorname{Conv}(R, T)$ since walls are convex. This contradicts $\sigma \not \subset \beta$.

We next introduce the interval determined by two spherical residues $R_{1}, R_{2}$ as the set $\left[R_{1}, R_{2}\right]$ consisting of those $\sigma \in \operatorname{Res}_{\mathrm{sph}}(X)$ such that $d\left(R_{1}, R_{2}\right)=d\left(R_{1}, \sigma\right)+d\left(\sigma, R_{2}\right)$.

Proposition 1.2. We have the following.

(i) The root-distance turns the set $\operatorname{Res}_{\mathrm{sph}}(X)$ into a (discrete) metric space.

(ii) Retractions on apartments do not increase the root-distance.

(iii) For all $R_{1}, R_{2} \in \operatorname{Res}_{\mathrm{sph}}(X)$, we have $\operatorname{Conv}\left(R_{1}, R_{2}\right)=\overline{\left[R_{1}, R_{2}\right]}$.

(iv) A set $\mathcal{R} \subset \operatorname{Res}_{\mathrm{sph}}(X)$ is convex if and only if it is closed and for all $R_{1}, R_{2} \in \mathcal{R}$, the interval $\left[R_{1}, R_{2}\right]$ is entirely contained in $\mathcal{R}$.

Before undertaking the proof, we record the following subsidiary fact which will be helpful in many arguments using induction on the root-distance. 
Lemma 1.3. Let $R_{1}, R_{2} \in \operatorname{Res}_{\mathrm{sph}}(X)$. Then the interval $\left[R_{1}, R_{2}\right]$ coincides with the pair $\left\{R_{1}, R_{2}\right\}$ if and only if $R_{1} \subset R_{2}$ or $R_{2} \subset R_{1}$ and no residue other than $R_{1}$ or $R_{2}$ is sandwiched between them.

Proof. The 'if' part is straightforward. Moreover, if $R_{1} \subset R_{2}$ and $R$ is a residue with $R_{1} \subset$ $R \subset R_{2}$, then $R \in\left[R_{1}, R_{2}\right]$. Therefore, it suffices to show that if $R_{1} \cap R_{2}$ is different from $R_{1}$ or $R_{2}$, then $] R_{1}, R_{2}\left[:=\left[R_{1}, R_{2}\right] \backslash\left\{R_{1}, R_{2}\right\}\right.$ is non-empty.

Consider the CAT(0) realisation $|X|$ of $X$ (see [Dav98]). Recall that the support of a point $x \in|X|$ is the unique minimal (i.e. lowest dimensional) spherical residue $R$ such that $x \in|R|$.

Assume first that there exist points $p_{1} \in\left|R_{2}\right|$ and $p_{2} \in\left|R_{2}\right|$ such that $p_{i}$ is supported by $R_{i}$ and that the geodesic segment $\left[p_{1}, p_{2}\right]$ is not entirely contained in $\left|R_{1}\right| \cup\left|R_{2}\right|$. Let then $x$ be a point of $\left[p_{1}, p_{2}\right] \backslash\left(\left|R_{1}\right| \cup\left|R_{2}\right|\right)$ and let $R$ denote the spherical residue supporting $x$. Clearly $R \neq R_{1}, R_{2}$. We claim that $R \in\left[R_{1}, R_{2}\right]$.

Let $A$ be an apartment containing $R_{1}$ and $R_{2}$. Then $R \subset A$. Since any root either contains $R$ or does not, we have $\Phi_{A}\left(R_{1}, R_{2}\right) \subset \Phi_{A}\left(R_{1}, R\right) \cup \Phi_{A}\left(R, R_{2}\right)$ and similarly with $R_{1}$ and $R_{2}$ interchanged. Thus it suffices to show that every root $\alpha$ containing $R$ but not $R_{2}$ also contains $R_{1}$ and vice-versa. But if $\alpha$ does not contain $R_{1}$, it does not contain $p_{1}$ since $p_{1}$ lies in the interior of $R_{1}$. Thus the wall $\partial \alpha$ does not separate $p_{1}$ from $p_{2}$, which contradicts the fact that $x \in|R| \subset|\alpha|$. This proves the claim.

Assume in a second case that for all points $p_{1}, p_{2}$ respectively supported by $R_{1}, R_{2}$, the geodesic segment $\left[p_{1}, p_{2}\right]$ lies entirely in $\left|R_{1}\right| \cup\left|R_{2}\right|$. Then $\left|R_{1}\right| \cap\left|R_{2}\right|$ is non-empty and $R:=R_{1} \cap R_{2}$ is thus a non-empty spherical residue. By the above, the residue $R$ is different from $R_{1}$ and $R_{2}$. We claim that $R \in\left[R_{1}, R_{2}\right]$. Let $A$ be an apartment containing $R_{1}$ and $R_{2}$; thus $R \subset A$. As before, it suffices to show that every root $\alpha$ of $A$ containing $R$ but not $R_{1}$ also contains $R_{2}$. If it didn't, then $-\alpha$ would contain two points $p_{1}, p_{2}$ respectively supported by $R_{1}, R_{2}$. In particular the geodesic segment $\left[p_{1}, p_{2}\right]$ is entirely contained in the interior of $-\alpha$ and, hence, it avoids $|R| \subset \alpha$. This is absurd since $|R|=\left|R_{1}\right| \cap\left|R_{2}\right|$.

Proof of Proposition 1.2. We start with the proof of (ii). Let $\rho$ be a retraction to some apartment $A$ and let $R_{1}, R_{2} \in \operatorname{Res}_{\mathrm{sph}}(X)$. We need to show that $d\left(\rho\left(R_{1}\right), \rho\left(R_{2}\right)\right) \leq d\left(R_{1}, R_{2}\right)$. We work by induction on $d\left(R_{1}, R_{2}\right)$, the result being trivial if $R_{1}=R_{2}$. Notice more generally that if $R_{1} \subset R_{2}$, then the restriction of $\rho$ to the pair $\left\{R_{1}, R_{2}\right\}$ is isometric, in which case the desired inequality holds trivially. We may therefore assume that $R_{1}$ and $R_{2}$ are not containing in one another. By Lemma 1.3, this implies that the open interval $] R_{1}, R_{2}[$ is non-empty. Let $R \in] R_{1}, R_{2}[$. Using the induction hypothesis, we deduce

$$
\begin{aligned}
d\left(R_{1}, R_{2}\right) & =d\left(R_{1}, R\right)+d\left(R, R_{2}\right) \\
& \geq d\left(\rho\left(R_{1}\right), \rho(R)\right)+d\left(\rho(R), \rho\left(R_{2}\right)\right) \\
& \geq d\left(\rho\left(R_{1}\right), \rho\left(R_{2}\right)\right),
\end{aligned}
$$

where the last inequality follows since any root of $A$ either contains $R$ or does not, whence $\Phi_{A}\left(R_{1}, R_{2}\right) \subset \Phi_{A}\left(R_{1}, R\right) \cup \Phi_{A}\left(R, R_{2}\right)$ and similarly with $R_{1}$ and $R_{2}$ interchanged.

(i) The only non-trivial point to check is the triangle inequality. We have just observed along the way that this inequality holds for triple of residues contained in a common apartment. The general case follows, using the fact that retractions do not increase distances.

(iii) We first use induction on $d\left(R_{1}, R_{2}\right)$ to prove that $\left[R_{1}, R_{2}\right] \subset \operatorname{Conv}\left(R_{1}, R_{2}\right)$.

Let thus $R \in\left[R_{1}, R_{2}\right]$. We shall show by induction on $d\left(R, R_{2}\right)$ that $R \in \operatorname{Conv}\left(R_{1}, R_{2}\right)$. 
Assume first that $] R, R_{2}[$ contains some spherical residue $T$. Then

$$
R \in\left[R_{1}, T\right] \subset \operatorname{Conv}\left(R_{1}, T\right) \subset \operatorname{Conv}\left(R_{1}, R_{2}\right),
$$

where the first inclusion follows from the induction on $d\left(R_{1}, R_{2}\right)$ and the second from the induction on $d\left(R, R_{2}\right)>d\left(T, R_{2}\right)$.

Assume now that $] R, R_{2}$ [ is empty. If $R \subset R_{2}$, then obviously $R \in \operatorname{Conv}\left(R_{1}, R_{2}\right)$. In view of Lemma 1.3 it only remains to deal with the case $R_{2} \subsetneq R$. In particular $d\left(R_{1}, R\right)<d\left(R_{1}, R_{2}\right)$, whence $\left[R_{1}, R\right] \subset \operatorname{Conv}\left(R_{1}, R\right)$ by induction. Since $\operatorname{Conv}\left(R_{1}, R\right)$ is closed, it contains $R_{2}$ and we deduce that some apartment $A$ contains $R_{1} \cup R_{2} \cup R$. Finally we observe that $\operatorname{Conv}\left(R_{1}, R\right)=$ $\operatorname{Conv}\left(R_{1}, R_{2}\right)$, since the fact that $R \in\left[R_{1}, R_{2}\right]$ implies that any root of $A$ which contains $R$ but not $R_{2}$ also contains $R_{1}$. Thus $R \in \operatorname{Conv}\left(R_{1}, R_{2}\right)$, which confirms the claim that $\left[R_{1}, R_{2}\right] \subset \operatorname{Conv}\left(R_{1}, R_{2}\right)$. In particular $\overline{\left[R_{1}, R_{2}\right]} \subset \operatorname{Conv}\left(R_{1}, R_{2}\right)$ since convex sets are closed.

Let now $x \in \operatorname{Conv}\left(R_{1}, R_{2}\right)$ and pick a maximal spherical residue $R \in \operatorname{Conv}\left(R_{1}, R_{2}\right)$ containing $x$. We claim that $R \in\left[R_{1}, R_{2}\right]$. Let thus $\alpha$ be a root containing $R$ but neither $R_{2}$ in some apartment $A$ containing $R_{1} \cup R_{2}$. If $R_{1} \not \subset \alpha$, then $\operatorname{Conv}\left(R_{1}, R_{2}\right) \subset-\alpha$ whence $R \subset \partial \alpha$. This

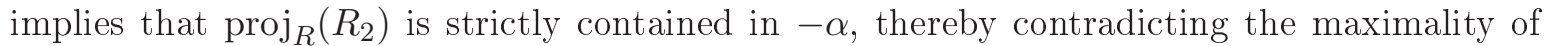
$R$. This shows that every root containing $R$ but not $R_{2}$ also contains $R_{1}$. A similar argument holds with $R_{1}$ and $R_{2}$ interchanged. This proves $R \in\left[R_{1}, R_{2}\right]$ as claimed. Thus $x \in \overline{\left[R_{1}, R_{2}\right]}$, which finishes the proof of (iii).

(iv) follows from (iii) since a set $\mathcal{R} \subset \operatorname{Res}_{\mathrm{sph}}(X)$ is convex if and only if it is closed and for all $R_{1}, R_{2} \in \mathcal{R}$, we have $\operatorname{Conv}\left(R_{1}, R_{2}\right) \subset \operatorname{Res}_{\mathrm{sph}}(X)$.

The following shows that the combinatorial projection of residues is canonically determined by the root-distance. In the special case of projections of chambers, the corresponding statements are well known.

Corollary 1.4. For all $R, T \in \operatorname{Res}_{\mathrm{sph}}(X)$, the projection $\operatorname{proj}_{R}(T)$ coincides with the unique maximal element of $[R, T]$ which contains $R$. It is also the unique spherical residue $\pi \supset R$ such that

$$
d(\pi, T)=\min \left\{d(\sigma, T) \mid \sigma \in \operatorname{Res}_{\mathrm{sph}}(X), \sigma \supset R\right\} .
$$

Proof. By Proposition 1.2(iii), the projection $\operatorname{proj}_{R}(T)$ is contained in some spherical residue $\pi \in[R, T]$. In particular $\pi$ is contained in $\operatorname{Conv}(R, T)$ and contains $R$. Therefore we have $\pi=\operatorname{proj}_{R}(T)$ by Lemma 1.1. Thus $\operatorname{proj}_{R}(T)$ is contained in the interval $[R, T]$ and the first assertion of the Corollary follows from Lemma 1.1 since $[R, T] \subset \operatorname{Conv}(R, T)$ by Proposition 1.2(iii).

The second assertion follows from arguments in the same vein than those which have been used extensively in this section. The details are left to the reader.

\section{Combinatorial compactifications}

2.1. Definition. The key ingredient for the construction of the combinatorial compactifications is the combinatorial projection. Given a residue $\sigma$, this projection is the map $\operatorname{proj}_{\sigma}: \operatorname{Ch}(X) \rightarrow \operatorname{St}(\sigma)$ which associates to a chamber $C$ the chamber of $\operatorname{St}(\sigma)$ which is nearest to $C$, see $[\mathrm{AB} 08, \S 4.9]$. As recalled in the previous section, the combinatorial projection may be extended to a map defined on the set of all residues of $X$. For our purposes, we shall focus on spherical residues and view the combinatorial projection as a map

$$
\operatorname{proj}_{\sigma}: \operatorname{Res}_{\mathrm{sph}}(X) \rightarrow \operatorname{St}(\sigma) .
$$


This allows one to define two maps

$$
\pi_{\mathrm{Ch}}: \operatorname{Ch}(X) \rightarrow \prod_{\sigma \in \operatorname{Res}_{1}(X)} \operatorname{St}(\sigma): C \mapsto\left(\sigma \mapsto \operatorname{proj}_{\sigma}(C)\right)
$$

and

$$
\pi_{\text {Res }}: \operatorname{Res}_{\mathrm{sph}}(X) \rightarrow \prod_{\sigma \in \operatorname{Res}_{\mathrm{sph}}(X)} \operatorname{St}(\sigma): R \mapsto\left(\sigma \mapsto \operatorname{proj}_{\sigma}(R)\right) .
$$

The above products are endowed with the product topology, where each star is a discrete set of residues. This allows one to consider the closure of the image of the above maps. In symbols, this yields the following definitions:

$$
\mathscr{C}_{1}(X)=\overline{\pi_{\mathrm{Ch}}(\operatorname{Ch}(X))} \quad \text { and } \quad \mathscr{C}_{\mathrm{sph}}(X)=\overline{\pi_{\operatorname{Res}}\left(\operatorname{Res}_{\mathrm{sph}}(X)\right)} .
$$

It is quite natural to consider the space $\overline{\pi_{\operatorname{Res}}(\operatorname{Ch}(X))}$ as well; in fact, we shall see in Proposition 2.12 below that this is equivariantly homeomorphic to $\mathscr{C}_{1}(X)$. We shall also see that $\mathscr{C}_{1}(X)$ may be identified to a closed subset of $\mathscr{C}_{\text {sph }}(X)$.

If the building $X$ is locally finite, then the star of each spherical residue is finite and hence the spaces $\mathscr{C}_{1}(X)$ and $\mathscr{C}_{\mathrm{sph}}(X)$ are then compact, and even metrizable since $\operatorname{Res}_{\mathrm{sph}}(X)$ is at most countable. The Aut $(X)$-action on $X$ extends in a canonical way to actions on $\mathscr{C}_{1}(X)$ and $\mathscr{C}_{\mathrm{sph}}(X)$ by homeomorphisms; the action induced by an element $g \in A u t(X)$ is given by

$$
g: \mathscr{C}_{\mathrm{sph}}(X) \rightarrow \mathscr{C}_{\mathrm{sph}}(X): f \mapsto\left(\sigma \mapsto g f\left(g^{-1} \sigma\right)\right) .
$$

Definition 2.1. The space $\mathscr{C}_{1}(X)$ and $\mathscr{C}_{\text {sph }}(X)$ are respectively called the minimal and the maximal combinatorial bordifications of $X$. When the building $X$ is locally finite, we shall use instead the term compactification.

This terminology is justified by the following.

Proposition 2.2. The maps $\pi_{\mathrm{Ch}}$ and $\pi_{\mathrm{Res}}$ are $G$-equivariant and injective; moreover, they have discrete images. In particular $\pi_{\mathrm{Ch}}$ and $\pi_{\mathrm{Res}}$ are homeomorphisms onto their images.

Proof. We argue only with $\pi_{\mathrm{Ch}}$, the case of $\pi_{\text {Res }}$ being similar. The equivariance is immediate. We focus on the injectivity. Let $C$ and $C^{\prime}$ be distinct chambers in $X$. There exists an apartment, say $A$, containing them both. These chambers are separated in $A$ by some wall $H$, so that the projections of $C$ and $C^{\prime}$ on every panel in $H$ cannot coincide. This implies that $\pi_{\mathrm{Ch}}(C) \neq \pi_{\mathrm{Ch}}\left(C^{\prime}\right)$ as desired.

Let now $\left(C_{n}\right)_{n \geq 0}$ be a sequence of chambers such that the sequence $\left(\pi_{\mathrm{Ch}}\left(C_{n}\right)\right)$ converges to $\pi_{\mathrm{Ch}}(C)$ for some $C \in \mathrm{Ch}(X)$. We have to show that $C_{n}=C$ for $n$ large enough. Suppose this is not the case. Upon extracting a subsequence, we may assume that $C_{n} \neq C$ for all $n$. Then there is some panel $\sigma_{n}$ in the boundary of $C$ such that $\operatorname{proj}_{\sigma_{n}}\left(C_{n}\right) \neq C$. Up to a further extraction, we may assume that $\sigma_{n}$ is independent of $n$ and denote by $\sigma$ the common value. Thus, we have $\operatorname{proj}_{\sigma}\left(C_{n}\right) \neq C$, which contradicts the fact that $\left(\pi_{\mathrm{Ch}}\left(C_{n}\right)\right)$ converges to $\pi_{\mathrm{Ch}}(C)$.

In the case when $X$ is locally finite, Proposition 2.2 implies that $\mathscr{C}_{1}(X)$ is indeed a compactification of the set of chambers of $X$ : In particular the discrete set $\pi_{\mathrm{Ch}}(\mathrm{Ch}(X))$ is open in $\mathscr{C}_{1}(X)$, which is thus indeed a compactification of $\mathrm{Ch}(X)$ in the locally finite case; a similar fact of course holds for $\mathscr{C}_{\mathrm{sph}}(X)$.

The elements $\mathscr{C}_{1}(X)$ and $\mathscr{C}_{\text {sph }}(X)$ are considered as functions which associate to every panel (resp. residue) a chamber (resp. residue) in the star of that panel (resp. residue). In view 
of Proposition 2.2 we may - and shall - identify $\operatorname{Ch}(X)$ and $\operatorname{Res}_{\mathrm{sph}}(X)$ to subsets of $\mathscr{C}_{1}(X)$ and $\mathscr{C}_{\text {sph }}(X)$. In particular, it makes sense to say that a sequence of chambers converges to a function in $\mathscr{C}_{1}(X)$.

We now take a closer look at the minimal bordification. The special case of a single apartment is straightforward:

Lemma 2.3. Let $f \in \mathscr{C}_{\mathrm{sph}}(X)$ and let $\left(R_{n}\right)$ and $\left(T_{n}\right)$ be sequences of spherical residues in a common apartment $A$ such that $\left(\pi_{\operatorname{Res}}\left(R_{n}\right)\right)$ and $\left(\pi_{\operatorname{Res}}\left(T_{n}\right)\right)$ both converge to $f$. Then for every half-apartment $\alpha$ in $A$, there is some $N \in \mathbb{N}$ such that either $R_{n} \cup T_{n} \subset \alpha$ for all $n>N$, or $R_{n} \cup T_{n} \subset-\alpha$ for all $n>N$, or $R_{n} \cup T_{n} \subset \partial \alpha$ for all $n>N$.

Conversely, let $\left(R_{n}\right)$ be a sequence of spherical residues of $A$ such that for every halfapartment $\alpha$ in $A$, there is some $N \in \mathbb{N}$ such that either $R_{n} \cup T_{n} \subset \alpha$ for all $n>N$, or $R_{n} \cup T_{n} \subset-\alpha$ for all $n>N$, or $R_{n} \cup T_{n} \subset \partial \alpha$ for all $n>N$. Then $\left(R_{n}\right)$ converges in $\mathscr{C}_{\mathrm{sph}}(A)$.

The same statements hold for sequences of chambers of $A$ and a point $f \in \mathscr{C}_{1}(X)$.

Proof. We deal only with the maximal bordification, the case of $\mathscr{C}_{1}(X)$ being similar but easier.

Let $H$ be a wall of $A$, let $\sigma \subset H$ and $C, C^{\prime}$ be the two chambers of $A$ containing $\sigma$. For any spherical residue $R$ of $A$, the projection $\operatorname{proj}_{\sigma}(R)$ coincides with $C$ (resp. $C^{\prime}$ ) if and only if $R$ lies on the same side of $H$ as $C$ (resp. $C^{\prime}$ ). It coincides with $\sigma$ itself if and only if $R$ lies on $H$. The result now follows from the very definition of the convergence in $\mathscr{C}_{\text {sph }}(X)$.

Let conversely $\left(R_{n}\right)$ be a sequence of spherical residues of $A$ which eventually remain on one side of every wall of $A$. Let $R \in \operatorname{Res}_{\text {sph }}(A)$. Let $\Phi$ denote the set of all roots $\alpha$ such that $R \subset \partial \alpha$ and $\left(R_{n}\right)$ eventually penetrates and remains in $\alpha$. Since $\Phi$ is finite, there is some $N$ such that $R_{n} \subset \bigcap_{\alpha \in \Phi} \alpha$ for all $n>N$. In particular $\operatorname{proj}_{R}\left(R_{n}\right) \subset \bigcap_{\alpha \in \Phi} \alpha$ for all $n>N$. It follows from Lemma 1.1 that $\operatorname{proj}_{R}\left(R_{n}\right)$ coincides with the unique maximal spherical residue contained in $\bigcap_{\alpha \in \Phi} \alpha$ and containing $R$. In particular, this is independent of $n>N$. Thus $\left(R_{n}\right)$ indeed converges in $\mathscr{C}_{\text {sph }}(A)$.

The subset of $\mathscr{C}_{1}(X)$ consisting of limits of sequences of chambers of an apartment $A$ is denoted by $\mathscr{C}_{1}(A)$, and $\mathscr{C}_{\operatorname{sph}}(A)$ is defined analogously. One verifies easily that this is consistent with the fact that $\mathscr{C}_{1}(A)\left(\right.$ resp. $\left.\mathscr{C}_{\text {sph }}(A)\right)$ also denotes the minimal (resp. maximal) bordification of the thin building $A$. However, it is not clear a priori that for every $f \in \mathscr{C}_{1}(X)$ belongs to $\mathscr{C}_{1}(A)$ for some apartment. Nevertheless, it turns out that this is indeed the case:

Proposition 2.4. The set $\mathscr{C}_{1}(X)$ is the union of $\mathscr{C}_{1}(A)$ taken over all apartments $A$. Similarly $\mathscr{C}_{\mathrm{sph}}(X)$ is covered by the union of $\mathscr{C}_{\mathrm{sph}}(A)$ over all apartments $A$.

This proposition is crucial in understanding the combinatorial compactifications, since it allows one to reduce many problems to the thin case. The proof requires some preparation and is thus postponed to $\S 2.6$

Example 2.5. Trees without leaves are buildings of type $D_{\infty}$. Panels in these trees are vertices and a sequence of chambers (i.e. edges) $\left(x_{n}\right)$ converges in the minimal bordification if the projection of $x_{n}$ on every vertex is eventually constant. It is easy to check that $\mathscr{C}_{1}(X)$ is isomorphic to the usual bordification of the tree, that is, to its set of ends. 
It is also possible to view a homogeneous tree of valency $r \geq 1$ as the Coxeter complex associated to the group $W=\left\langle s_{1}, \ldots, s_{r} \mid s_{1}^{2}, \ldots, s_{r}^{2}\right\rangle$. The panels are then the middles of the edges, a chamber is a vertex with all the half-edges which are incident to it. From this viewpoint as well, the combinatorial bordification coincides with the visual one.

Example 2.6. (This example may be compared to [GR06, 6.3.1]) Let us consider an apartment $A$ of type $\tilde{A}_{2}$. It is a Euclidean plane, tessellated by regular triangles. We know by Lemma 2.3 that we can characterize the points $\xi \in \mathscr{C}_{1}(A)$ by the sets of roots $\Phi(\xi)$ associated to them. We may distinguish several types of boundary points. Let us choose some root basis $\left\{a_{1}, a_{2}\right\}$ in the vectorial system of roots. Then there is a point $\xi \in \mathscr{C}_{1}(A)$ defined by $\Phi(\xi)=\left\{a_{1}+\right.$ $\left.k, a_{2}+l \mid k, l \in \mathbb{Z}\right\}$. There are six such points, which correspond to a choice of positive roots, i.e. to a Weyl chamber in $A$. The sequences of (affine) chambers that converge to these points are the sequences that eventually stay in a given sector, but whose distances to each of the two walls in the boundary of this sector tend to infinity.

There is also another category of boundary points, which corresponds to sequence of chambers that stay in a given sector, but stay at bounded distance of one of the two walls defining this sector. With a choice of $a_{1}$ and $a_{2}$ as before, these are points associated to set of roots of the form $\left\{a_{1}+k, a_{2}+l \mid k, l \in \mathbb{Z}, k \leq k_{0}\right\}$. As $k_{0}$ varies, we get a 'line' of such points, and there are six such lines.

When $X$ is a building of type $\tilde{A}_{2}$, by Proposition 2.4, we can always write a point in the boundary of $X$ as a point in the boundary of some apartment of $X$. Thus, the previous description applies to general points of the bordification.

Example 2.7. Let $W$ be a Fuchsian Coxeter group, that is, whose Coxeter complex is a tessellation of the hyperbolic plane. Assume the action of $W$ on the hyperbolic plane is cocompact. As in the previous example we shall content ourselves with a description of the combinatorial compactification of some apartment $A$. In order to do so, we shall use the visual boundary $\partial_{\infty} \Sigma \simeq S^{1}$. If there is a point $\xi$ of this boundary towards which no wall is pointing, then we can associate to it a point of the combinatorial compactification, just by taking the roots that contain a sequence of points converging to $\xi$. If we have a point of the boundary towards which $n$ walls are pointing, we associate to it $n+1$ points in $\mathscr{C}_{1}(A)$, whose positions are defined in relations to the roots which have these $n$ walls as a boundary.

Moreover, let us remark that the set of limit points of walls is dense in the boundary of the hyperbolic plane. To prove that, it is enough to check that the action of $W$ on $S^{1}$ is minimal (all its orbits are dense). Using [GdlH90, Corollary 26], the action of $W$ on its limit set $L(W)$ is minimal, and by [Kat92, Theorem 4.5.2], the limit set $L(W)$ is in fact $S^{1}$.

Therefore, if $\xi$ and $\xi^{\prime}$ are two regular points (i.e. towards which no wall is pointing), then $\xi$ and $\xi^{\prime}$ are separated by some wall. In particular, we see that the construction we have just made always yields different points. Therefore, the compactification $\mathscr{C}_{1}(A)$ is a refinement of the usual boundary.

Example 2.8. If $X=X_{1} \times X_{2}$ is a product of buildings, then a chamber of $X$ is just the product of a chamber in $X_{1}$ and a chamber in $X_{2}$, and the projection on another chamber is just the product of the projections in $X_{1}$ and $X_{2}$. Therefore, the combinatorial compactification $\mathscr{C}_{\text {sph }}(X)$ is the product $\mathscr{C}_{\text {sph }}\left(X_{1}\right) \times \mathscr{C}_{\text {sph }}\left(X_{2}\right)$. 
2.2. Projecting from infinity. In this section, we show that any function $f \in \mathscr{C}_{1}(X)$ admits a projection on every spherical residue of $X$. This allows us to define the embedding of $\mathscr{C}_{1}(X)$ into $\mathscr{C}_{\text {sph }}(X)$ alluded to above.

Let thus $\xi \in \mathscr{C}_{1}(X)$ and $R$ be any residue. Recall that $R$ is a building [Ron89, Theorem 3.5]. We define the projection of $\xi$ to $R$, denoted $\operatorname{proj}_{R}(\xi)$, to be the restriction of $\xi$ to $\operatorname{Res}_{1}(R)$. In the special case when $R$ is a panel, the set $\operatorname{Res}_{1}(R)$ is a singleton and the function $\operatorname{proj}_{R}(\xi)$ may therefore be identified with a chamber which coincides with $\xi(R)$.

Similarly, given $\xi \in \mathscr{C}_{\mathrm{sph}}(X)$ we define $\operatorname{proj}_{R}(\xi)$ as the restriction of $\xi$ to $\operatorname{Res}_{\mathrm{sph}}(R)$.

The next statement ensures that the definition of $\operatorname{proj}_{R}$ is meaningful.

Lemma 2.9. Let $\left(C_{n}\right)$ be a sequence of chambers converging to $\xi \in \mathscr{C}_{1}(X)$ and let $R$ be a residue in $X$. The sequence of projections $\left(\operatorname{proj}_{R}\left(C_{n}\right)\right)$ converges to an element $\operatorname{proj}_{R}(\xi) \in$ $\prod_{\sigma \in \operatorname{Res}_{1}(R)} \operatorname{St}(\sigma)$. In particular $\operatorname{proj}_{R}(\xi)$ is an element of $\mathscr{C}_{1}(R)$.

Similarly, any sequence $\left(R_{n}\right)$ converging to some $\eta \in \mathscr{C}_{\mathrm{sph}}(X)$ yields a sequence $\left(\operatorname{proj}_{R}\left(R_{n}\right)\right)$ which converges to some element of $\mathscr{C}_{\mathrm{sph}}(R)$ which is denoted by $\operatorname{proj}_{R}(\eta)$.

Proof. We focus on the minimal compactification; the maximal one is similar.

It is enough to prove the very first point. By definition of the convergence in $\mathscr{C}_{1}(X)$, for every panel $\sigma \supset R$, there exists some integer $N$ depending on $\sigma$ such that for $n>N$, $\operatorname{proj}_{\sigma}\left(C_{n}\right)=\xi(\sigma)$. Moreover we have

$$
\operatorname{proj}_{R}\left(\operatorname{proj}_{\sigma}\left(C_{n}\right)\right)=\operatorname{proj}_{R}(\xi(\sigma))=\xi(\sigma) .
$$

Now $\operatorname{proj}_{\sigma}\left(C_{n}\right)$ coincides with $\operatorname{proj}_{\sigma}\left(\operatorname{proj}_{R}\left(C_{n}\right)\right)$. Hence, for $n>N$, we have

$$
\operatorname{proj}_{\sigma}\left(\operatorname{proj}_{R}\left(C_{n}\right)\right)=\xi(\sigma),
$$

which is equivalent to saying that $\left(\operatorname{proj}_{R}\left(C_{n}\right)\right)$ converges to $\operatorname{proj}_{R}(\xi)$.

In Lemma 2.9 , if the residue $R$ is spherical then for any $\xi \in \mathscr{C}_{1}(X)$, the projection $\operatorname{proj}_{R}(\xi)$ may be identified with a chamber of $R$. Similarly, for any $\eta \in \mathscr{C}_{\text {sph }}(X)$, the projection $\operatorname{proj}_{R}(\eta)$ may be identified with a residue in $\operatorname{St}(R)$.

Let now $C$ be a chamber and $\xi$ be a point in the boundary of $\mathscr{C}_{1}(X)$. As $\xi$ is not equal to $C$, there exists some panel in the boundary of $C$ on which the projection of $\xi$ is different from the projection of $C$. Let $I$ be the set of all such panels, and consider the residue $R$ of type $I$ containing $C$.

Lemma 2.10. The residue $R$ is spherical and $\operatorname{proj}_{R}(\xi)$ is a chamber opposite $C$ in $R$.

Proof. Let $\left(C_{n}\right)$ be a sequence converging to $\xi$. By Lemma $2.9,\left(\operatorname{proj}_{R}\left(C_{n}\right)\right)$ converges to $\operatorname{proj}_{R}(\xi)$. Therefore, the projection of $\operatorname{proj}_{R}\left(C_{n}\right)$ on the panels adjacent to $C_{n}$ is eventually the same as the projection of $\operatorname{proj}_{R}(\xi)$, that is, of $\xi$. In other words, the projection of $C_{n}$ on every panel of $R$ in the boundary of $C$ is always different from $C$. By [Bro89, IV.6, Lemma 3], this implies that $R$ is spherical and that $\operatorname{proj}_{R}\left(C_{n}\right)$ is opposite to $C$. As $\operatorname{proj}_{R}\left(C_{n}\right)$ converges to $\operatorname{proj}_{R}(\xi)$, this implies that $\operatorname{proj}_{R}(\xi)$ is opposite to $C$ in $R$.

Definition 2.11. The residue $R$ defined as above is called the residual projection of $\xi$ on $C$.

Proposition 2.12. There is a $G$-equivariant continuous injective map $\mathscr{C}_{1}(X) \rightarrow \mathscr{C}_{\mathrm{sph}}(X)$. Its image coincides with the closure of $\mathrm{Ch}(X)$ in $\mathscr{C}_{\mathrm{sph}}(X)$. 
Proof. As pointed out before, the set $\operatorname{Res}_{\mathrm{sph}}(X)$ may be identified with a subset of $\mathscr{C}_{\mathrm{sph}}(X)$ via the map $\pi_{\text {Res. }}$. In particular we may view $\mathrm{Ch}(X)$ as a subset of $\mathscr{C}_{\mathrm{sph}}(X)$. Projections to residues allows one to extend this inclusion to a well defined map $\mathscr{C}_{1}(X) \rightarrow \mathscr{C}_{\text {sph }}(X)$. The fact that it is injective and continuous is straightforward to check; the details are left to the reader.

In view of this Proposition, we may identify $\mathscr{C}_{1}(X)$ to a closed subset of $\mathscr{C}_{\mathrm{sph}}(X)$. The fact that $\mathscr{C}_{1}(X) \cap \operatorname{Res}_{\mathrm{sph}}(X)$ coincides with $\mathrm{Ch}(X)$ motivates the following definition.

Definition 2.13. A point of $\mathscr{C}_{\mathrm{sph}}(X)$ which belongs to $\mathscr{C}_{1}(X)$ is called a chamber. If it does not belong to $\mathrm{Ch}(X)$, we say that it is a chamber at infinity.

2.3. Extending the notion of sectors to arbitrary buildings. The notion of sectors is crucial in analysing the structure of Euclidean buildings. In this section we propose a generalisation of this notion to arbitrary buildings. This will turn out to be a crucial tool for the study of the combinatorial bordifications.

Let $x \in \operatorname{Res}_{\mathrm{sph}}(X)$ be a spherical residue and $\left(R_{n}\right)$ be a sequence of spherical residues converging to some $\xi \in \mathscr{C}_{\operatorname{sph}}(X)$. In order to simplify the notation, we shall denote the sequence $\left(R_{n}\right)$ by $\underline{R}$. For any integer $k \geq 0$ we set

$$
Q_{k}=\bigcap_{n \geq k} \operatorname{Conv}\left(x, R_{n}\right)
$$

and

$$
Q(x, \underline{R})=\bigcup_{k \geq 0} Q_{k} .
$$

Since $Q_{k}$ is contained in an apartment and since $Q_{k} \subset Q_{k+1}$ for all $k$, it follows from standard arguments that $Q(x, \underline{R})$ is contained in some apartment of $X$ (compare [Tit81, $\S 3.7 .4]$ or [Ron89, Theorem 3.6]).

Remark 2.14. Retain the same notation as before and let $y$ be a spherical residue. If $y \subset$ $Q(x, \underline{R})$, then we have $Q(y, \underline{R}) \subset Q(x, \underline{R})$.

Proposition 2.15. Let $\left(R_{n}\right)=\underline{R}$ be a sequence of spherical residues converging to $\xi \in$ $\mathscr{C}_{\mathrm{sph}}(X)$ and let $x \in \operatorname{Res}_{\mathrm{sph}}(X)$.

(i) The set $Q(x, \underline{R})$ only depends on $x$ and $\xi$, and not on the sequence $\underline{R}$.

(ii) $Q(x, \underline{R})$ may be characterised as the smallest subcomplex $P$ of $X$ containing $x$ and such that if $R$ is a spherical residue in $P$, then for every $\sigma \in \operatorname{St}(R)$, the projection $\operatorname{proj}_{\sigma}(\xi)$ is again in $P$.

Proof. Clearly (i) is a consequence of (ii).

Set $Q:=Q(x, \underline{R})$ and define $Q^{\prime}$ to be the $\xi$-convex hull of $x$. By definition, this means that $Q^{\prime}$ is the minimal set of spherical simplices satisfying the following three conditions:

- $x \in Q^{\prime}$.

- $Q^{\prime}$ is closed.

- For any spherical residue $\sigma \subset Q^{\prime}$ we have $\operatorname{proj}_{\sigma}(\xi) \subset Q^{\prime}$.

We have to show that $Q=Q^{\prime}$. To this end, let $\mathcal{V}$ denote the collection of all subsets of $\operatorname{Res}_{\text {sph }}(X)$ satisfying the above three conditions. Thus $Q^{\prime}=\bigcap \mathcal{V}$.

By definition, for each $k \geq 0$ the subcomplex $Q_{k}$ is convex, hence closed, and contains $x$. Therefore the same holds true for $Q$. We claim that $Q \in \mathcal{V}$. Indeed, for any $\sigma \in \operatorname{Res}_{\operatorname{sph}}(X)$ the 
projection $\operatorname{proj}_{\sigma}(\xi)$ coincides with $\operatorname{proj}_{\sigma}\left(R_{n}\right)$ for $n$ large enough (see Lemma 2.9). Therefore, for any $\sigma \in Q$ there exists a sufficiently large $k$ such that $\operatorname{proj}_{\sigma}(\xi)=\operatorname{proj}_{\sigma}\left(R_{n}\right) \subset \operatorname{Conv}\left(x, R_{n}\right)$ for all $n>k$. Thus $\operatorname{proj}_{\sigma}(\xi) \subset Q_{k} \subset Q$, which confirms that $Q \in \mathcal{V}$. In particular we deduce that $Q^{\prime} \subset Q$.

Let now $R$ be a spherical residue in $Q$. We shall show by induction on the root-distance of $R$ to $x$ that $R \subset Q^{\prime}$.

Assume first that $x \supset R$. Then $R \in Q^{\prime}$ since $Q^{\prime}$ is closed and contains $x$. Assume next that $x \subset R$. As $R \in Q$, we have $R \in \operatorname{Conv}\left(x, R_{n}\right)$ for any $n$ large enough. Since $\operatorname{proj}_{x}\left(R_{n}\right)$ is the largest residue contained in $\operatorname{Conv}\left(x, R_{n}\right)$ and containing $x$ (see Lemma 1.1), we have $R \subset \operatorname{proj}_{x}\left(R_{n}\right)$. It follows that $R \in Q^{\prime}$ since $Q^{\prime}$ is closed and since for large $n$ we have $\operatorname{proj}_{x}\left(R_{n}\right)=\operatorname{proj}_{x}(\xi) \in Q^{\prime}$.

In view of Lemma 1.3, we may now assume that the interval $[x, R]$ is non-empty and contains some spherical residue $x^{\prime}$. By induction $x^{\prime} \in Q^{\prime}$. Let $n$ be large enough so that $R \in \operatorname{Conv}\left(x, R_{n}\right)$. We have $x^{\prime} \in[x, R] \subset \operatorname{Conv}(x, R)$ by Proposition 1.2(iii). Thus, in an apartment containing $\operatorname{Conv}\left(x, R_{n}\right)$, any root containing $x$ and $R$ also contains $x^{\prime}$. One deduces that $R \in \operatorname{Conv}\left(x^{\prime}, R_{n}\right)$ for all large $n$. In particular $R \in Q\left(x^{\prime}, \underline{R}\right)$. By induction, we deduce that $R$ belongs to the $\xi$-convex hull of $x^{\prime}$, which we denote by $Q^{\prime}\left(x^{\prime}\right)$. Since $x^{\prime} \in Q^{\prime}$, we have $Q^{\prime}\left(x^{\prime}\right) \subset Q^{\prime}$ whence $R \in Q^{\prime}$ as desired.

Proposition 2.15 shows that $Q(x, \underline{R})$ depends only on $\xi=\lim \underline{R}$. It therefore makes sense to write $Q(x, \xi)$ instead of $Q(x, \underline{R})$.

Definition 2.16. The set $Q(x, \xi)$ is called the combinatorial sector, or simply the sector, pointing towards $\xi$ and based at $x$.

Remark 2.17. In the affine case, sectors in the classical sense are also combinatorial sectors in the sense of the preceding definition (see example 2.21). However, the converse is not true in general.

The following shows that the sector $Q(x, \xi)=Q\left(x,\left(R_{n}\right)\right)$ should be thought of as the pointwise limit of $\operatorname{Conv}\left(x, R_{n}\right)$ as $n$ tends to infinity:

Corollary 2.18. Let $x \in \operatorname{Res}_{\mathrm{sph}}(X)$ and $\left(R_{n}\right)$ be a sequence of spherical residues converging to some $\xi \in \mathscr{C}_{\mathrm{sph}}(X)$. For any finite subset $F \subset \operatorname{Res}_{\mathrm{sph}}(X)$ there is some $N \geq 0$ such that for any $n>N$, the respective intersections of the sets $Q(x, \xi)$ and $\operatorname{Conv}\left(x, R_{n}\right)$ with $F$ coincide.

Proof. It suffices to show that for each $y \in F$, either $y \subset \operatorname{Conv}\left(x, R_{n}\right)$ for all large $n$, or $y \not \subset \operatorname{Conv}\left(x, R_{n}\right)$ for all large $n$.

If $y \subset Q(x, \xi)$, this follows at once from the definition of $Q(x, \xi)$.

Assume now that $y$ is not contained in $Q(x, \xi)$. We claim that there is some $N>0$ such that $y \not \subset \operatorname{Conv}\left(x, R_{n}\right)$ for all $n>N$. Indeed, in the contrary case for each $n>0$ there is some $\varphi(n)>n$ such that $y$ is contained in $\operatorname{Conv}\left(x, R_{\varphi(n)}\right)$. Therefore we have $y \subset \bigcap_{n>0} \operatorname{Conv}\left(x, R_{\varphi(n)}\right)$. Since $\left(R_{\varphi(n)}\right)$ converges to $\xi$ it follows from Proposition 2.15 that $Q\left(x,\left(R_{\varphi(n)}\right)\right)=Q(x, \xi)$ and we deduce that $y \subset Q(x, \xi)$, which is absurd.

The following interpretation of the projection from infinity shows in particular that for all $x \in \operatorname{Res}_{\mathrm{sph}}(X)$ and $\xi \in \mathscr{C}_{\mathrm{sph}}(X)$, the projection $\operatorname{proj}_{x}(\xi)$ is canonically determined by the sector $Q(x, \xi)$, viewed as a set of spherical residues. 
Corollary 2.19. For all $x \in \operatorname{Res}_{\mathrm{sph}}(X)$ and $\xi \in \mathscr{C}_{\mathrm{sph}}(X)$, the projection $\operatorname{proj}_{x}(\xi)$ coincides with the unique maximal residue containing $x$ and contained in $Q(x, \xi)$.

Proof. Follows from Lemma 1.1 and Corollary 2.18.

Example 2.20. Let $X$ be a tree. It has been seen in example 2.5 that $\mathscr{C}_{1}(X)$ coincides with the visual compactification $X \cup \partial_{\infty} X$. If $\xi \in \partial_{\infty} X$, then $Q(x, \xi)$ is the half-line starting from $x$ and pointing towards $\xi$.

Example 2.21. Let $X$ be a building of type $\tilde{A}_{2}$. As explained in example 2.6, there are several type of boundary points. The sectors pointing towards different types of boundary points have different shapes. Furthermore, there are two possible orientations for chambers, which give also different shapes to the sectors.

Let $\xi_{1}$ be the point of the boundary which corresponds, in a given apartment $A$, to a set of roots $\Phi_{A}\left(\xi_{1}\right)=\left\{a_{1}+k, a_{2}+l \mid k, l \in \mathbb{Z}\right\}$. Let $x$ be a chamber in $A$. To determine $Q\left(x, \xi_{1}\right)$, we have to consider the roots in the direction of $a_{1}$ and $a_{2}$ that contain $x$. We then have two possibilities for $Q\left(x, \xi_{1}\right)$, according to the orientation of $x$. If $x$ is oriented 'towards $\xi_{1}$ ', then $Q\left(x, \xi_{1}\right)$ is the classical sector based at $x$ and pointing towards $\xi_{1}$. Otherwise, $Q\left(x, \xi_{1}\right)$ is a troncated sector. These two possibilities are described on Figure 1.

Now, let $\xi_{2}$ be the point in the boundary of $A$ determined by $\Phi_{A}\left(\xi_{2}\right)=\left\{a_{1}+k, a_{2}+l \mid k, l \in\right.$ $\left.\mathbb{Z}, k \leq k_{0}\right\}$. The sector $Q\left(x, \xi_{2}\right)$ is determined in the same way as $Q\left(x, \xi_{1}\right)$, but we now have to stop at the root $a_{1}+k_{0}$. Thus, we get a 'half-strip' which goes from $x$, stops at the root $a_{1}+k_{0}$, and is in the direction of $a_{2}$. Once again, the precise shape of this half-strip depends on the orientation of $x$. These two possibilites are described on Figure 2.

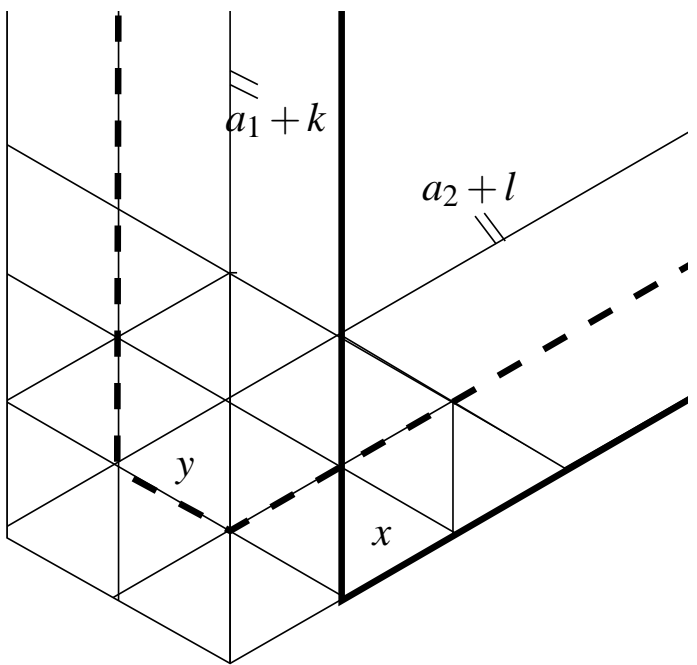

Figure 1. $Q\left(x, \xi_{1}\right)$ and $Q\left(y, \xi_{1}\right)$, where $\Phi\left(\xi_{1}\right)=$ $\left\{a_{1}+k, a_{2}+l \mid k, l \in \mathbb{Z}\right\}$

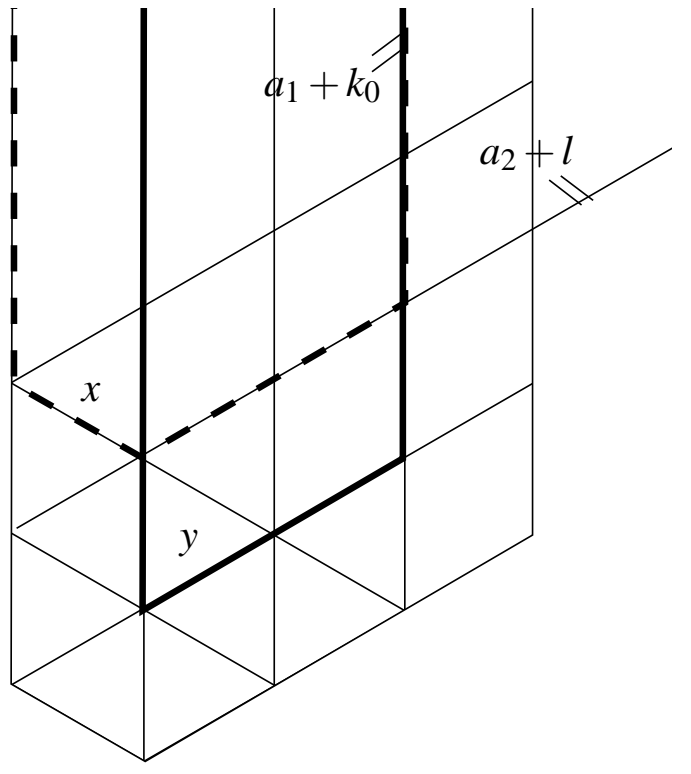

Figure 2. $Q\left(x, \xi_{2}\right)$ and $Q\left(y, \xi_{2}\right)$, where $\Phi\left(\xi_{2}\right)=$ $\left\{a_{1}+k, a_{2}+l \mid k, l \in \mathbb{Z}, k \leq\right.$ $\left.k_{0}\right\}$ 
2.4. Sectors and half-apartments. The use of sectors will eventually allow us to study the combinatorial compactifications of $X$ by looking at one apartment at a time. The first main goal is to obtain a proof of Proposition 2.4. Not surprisingly, retractions provide an important tool.

Lemma 2.22. Let $x \in \mathrm{Ch}(X)$ and $\left(R_{n}\right)$ be a sequence of spherical residues converging to some $\xi \in \mathscr{C}_{\mathrm{sph}}(X)$. Let $A$ be an apartment containing the sector $Q(x, \xi)$. Then we have

$$
Q(x, \xi)=\bigcup_{k \geq 0} \bigcap_{n \geq k} \operatorname{Conv}\left(x, \rho_{A, x}\left(R_{n}\right)\right) .
$$

Proof. Let $Q_{k}=\bigcap_{n \geq k} \operatorname{Conv}\left(x, R_{n}\right), Q_{k}^{\prime}=\bigcap_{n \geq k} \operatorname{Conv}\left(x, \rho_{A, x}\left(R_{n}\right)\right), Q=Q(x, \xi)=\bigcup Q_{k}$ and $Q^{\prime}=\bigcup Q_{k}^{\prime}$. We must show that $Q=Q^{\prime}$. Since these are both (closed and) convex and contain the chamber $x$, it suffices to show that $\operatorname{Ch}(Q)=\operatorname{Ch}\left(Q^{\prime}\right)$.

Fix $k \geq 0$. Let $C \in \operatorname{Ch}\left(Q_{k}\right)$ and let $n \geq k$. Then $C$ belongs to a minimal gallery from $x$ to a chamber containing $R_{n}$. Since $C \subset A$, the retraction $\rho_{A, x}$ fixes $C$ and hence $C$ belongs to a minimal gallery from $x$ to a chamber containing $\rho_{A, x}\left(R_{n}\right)$. This shows that $\operatorname{Ch}\left(Q_{k}\right) \subset \operatorname{Ch}\left(Q_{k}^{\prime}\right)$. Therefore we have $\operatorname{Ch}(Q) \subset \operatorname{Ch}\left(Q^{\prime}\right)$.

Suppose for a contradiction that there exists some $C \in \mathrm{Ch}\left(Q^{\prime}\right) \backslash \mathrm{Ch}(Q)$. Choose $C$ at minimal possible distance to $\operatorname{Ch}(Q)$. Thus $C$ is adjacent to some chamber $C^{\prime} \in \operatorname{Ch}(Q)$. Let $\sigma=C \cap C^{\prime}$ be the panel shared by $C$ and $C^{\prime}$ and $\alpha$ be the half-apartment containing $C^{\prime}$ and such that $\partial \alpha$ contains $\sigma$. Since $\sigma \subset Q$, we have $\operatorname{proj}_{\sigma}(x) \subset Q$, whence $\operatorname{proj}_{\sigma}(x)=C^{\prime}$ and $x \subset \alpha$.

Let now $k$ be such that $C \subset \operatorname{Conv}\left(x, \rho_{A, x}\left(R_{n}\right)\right)$ for all $n \geq k$ and let $C_{n}$ denote the unique element of $\operatorname{Conv}\left(x, R_{n}\right)$ such that $\rho_{A, x}\left(C_{n}\right)=C$. Each $C_{n}$ contains the panel $\sigma$ and we have $\operatorname{proj}_{\sigma}\left(R_{n}\right)=C_{n}$. Therefore, we deduce that $\xi(\sigma)=C_{n}$ (see Lemma 2.9). Since $Q \subset A$, this implies that $C_{n} \subset A$ by Proposition 2.15. Therefore we have $C=\rho_{A, x}\left(C_{n}\right)=C_{n}$, whence $C \subset \operatorname{Conv}\left(x, R_{n}\right)$ for all $n \geq k$. This implies that $C$ is contained in $Q$, which is absurd.

Lemma 2.23. Let $x \in \mathrm{Ch}(X)$ and $\left(R_{n}\right)$ be a sequence of spherical residues converging to some $\xi \in \mathscr{C}_{\mathrm{sph}}(X)$. Let $A$ be an apartment containing the sector $Q(x, \xi)$. Then for any chamber $C \in \operatorname{Ch}(A)$, we have $Q(C, \xi) \subset A$. Moreover there exists $k \geq 0$ such that $\rho_{A, C}\left(R_{n}\right)=\rho_{A, x}\left(R_{n}\right)$ for all $n>k$.

Proof. By connexity of $A$, it suffices to prove that for any chamber $C$ adjacent to $x$, the sector $Q(C, \xi)$ is contained in $A$ and furthermore that $\rho_{A, C}\left(R_{n}\right)=\rho_{A, x}\left(R_{n}\right)$ for all sufficiently large $n$. Let $\sigma=x \cap C$ be the panel separating $x$ from $C$.

Since $\sigma \subset Q(x, \xi)$, Proposition 2.15 implies that $\xi(\sigma)$ is contained in $A$. The only possible values for $\xi(\sigma)$ are thus $x, C$ and $\sigma$. We treat these three cases successively.

If $\xi(\sigma)=C$ then $C \subset Q(x, \xi)$, whence $Q(C, \xi) \subset A$ by Remark 2.14. The desired claims follow by definition.

If $\xi(\sigma)=x$ then $x \in \operatorname{Conv}\left(C, R_{n}\right)$ for $n$ sufficiently large. Thus there is a minimal gallery from $C$ to a chamber containing $R_{n}$ via $x$ and it follows that $\rho_{A, C}\left(R_{n}\right)=\rho_{A, x}\left(R_{n}\right)$ since $x \in \operatorname{Ch}(A)$. The fact that $Q(C, \xi)$ is contained in $A$ now follows from Lemma 2.22.

If $\xi(\sigma)=\sigma$, pick a large enough $n$ so that $\operatorname{proj}_{\sigma}\left(R_{n}\right)=\sigma$. Let $A_{n}$ be an apartment containing $x \cup R_{n}$. Then $R_{n}$ lies on a wall $H_{n}$ of $A_{n}$ containing $\sigma$, and the convex hull $\operatorname{Conv}\left(x, R_{n}\right)$ lies entirely on one side of $H_{n}$; we call the latter half-apartment $\alpha$. Since the chamber $C$ contains the panel $\sigma \subset H_{n}$, there is a half-apartment $\beta$ bounding $H_{n}$ and containing 
$C$. Therefore, upon replacing $A_{n}$ by $\alpha \cup \beta$, there is no loss of generality in assuming that $C$ is contained in $A_{n}$. It follows readily that $\rho_{A, C}\left(R_{n}\right)=\rho_{A, x}\left(R_{n}\right)$. As in the previous case, the fact that $Q(C, \xi)$ is contained in $A$ follows from Lemma 2.22 .

The following is an analogue of Lemma 2.3 in the non-thin case.

Lemma 2.24. Let $x \in \mathrm{Ch}(X),\left(R_{n}\right)$ be a sequence of spherical residues converging to some $\xi \in \mathscr{C}_{\mathrm{sph}}(X)$ and $A$ be an apartment containing the sector $Q(x, \xi)$. Set $R_{n}^{\prime}=\rho_{A, x}\left(R_{n}\right)$. For any half-apartment $\alpha$ of $A$, there is some $N$ such that for all $n>N$ we have $R_{n}^{\prime} \subset \alpha$ or $R_{n}^{\prime} \subset-\alpha$.

Proof. Let $C, C^{\prime}$ be the chambers of $A$ such that $C \cap C^{\prime}=\sigma$. Let also $\alpha$ be the half-apartment containing $C$ but not $C^{\prime}$. Assume that the sequence $\left(R_{n}^{\prime}\right)$ possesses two subsequences $R_{\varphi(n)}^{\prime}$ and $R_{\psi(n)}^{\prime}$ such that $R_{\varphi(n)}^{\prime}$ is strictly contained in $\alpha$ and $R_{\psi(n)}^{\prime}$ is strictly contained in $-\alpha$. Then $\operatorname{Conv}\left(C, R_{\psi(n)}^{\prime}\right)$ contains $C^{\prime}$ for all $n$ while $\operatorname{Conv}\left(C, R_{\varphi(n)}^{\prime}\right)$ does not. This contradicts Lemma 2.22, thereby showing that the sequence $R_{n}^{\prime}$ eventually remains on one side of the wall $\partial \alpha$.

Definition 2.25. Let $\Phi_{A}(\xi)$ denote the set of all half-apartments $\alpha$ of $A$ such that the sequence $\left(R_{n}^{\prime}\right)$ eventually lies in $\alpha$. In view of Lemma 2.23, this set is independent of $x \in \operatorname{Ch}(A)$, but depends only on $A$ and $\xi$.

One should think of the elements of $\Phi_{A}(\xi)$ as half-apartments 'containing' the point $\xi$. Notice that if $\xi=R$ is a residue, then $\Phi_{A}(\xi)$ is nothing but the set of those half-apartments which contain $R$.

Notice that two opposite roots $\alpha,-\alpha$ might be both contained in $\Phi_{A}(\xi)$; in view of Lemma 2.24, this happens if and only if the residue $\left(R_{n}^{\prime}\right)$ lies on the wall $\partial \alpha$ for all sufficiently large $n$.

Lemma 2.26. Let $x \in \mathrm{Ch}(X)$ and $\left(R_{n}\right)$ be a sequence of spherical residues converging to some $\xi \in \mathscr{C}_{\mathrm{sph}}(X)$. Let $A$ be an apartment containing the sector $Q(x, \xi)$. Then the sequence $\left(\rho_{A, x}\left(R_{n}\right)\right)$ converges in $\mathscr{C}_{\mathrm{sph}}(A)$ and its limit coincides with the restriction of $\xi$ to $\operatorname{Res}_{\mathrm{sph}}(A)$.

Furthermore, for any $\xi^{\prime} \in \mathscr{C}_{\mathrm{sph}}(A)$, we have $\xi^{\prime}=\xi$ if and only if $\Phi_{A}\left(\xi^{\prime}\right)=\Phi_{A}(\xi)$.

Proof. Let $R \in \operatorname{Res}_{\mathrm{sph}}(A)$ and $H(R)$ denote the (finite) set of all walls containing $R$. By Lemma 2.24, there is some $N$ such that $R_{n}^{\prime}$ remains on one side of each wall in $H(R)$ for all $n>N$. The fact that the sequence $\left(R_{n}^{\prime}\right)$ converges to some $\xi^{\prime} \in \mathscr{C}_{\mathrm{sph}}(A)$ thus follows from Lemma 2.3. By construction we have $\Phi_{A}\left(\xi^{\prime}\right)=\Phi_{A}(\xi)$. All it remains to show is thus that $\xi$ and $\xi^{\prime}$ coincide.

We first show that they coincide on panels. Let thus $\sigma \subset A$ be a panel and $C, C^{\prime} \in \operatorname{Ch}(A)$ be such that $C \cap C^{\prime}=\sigma$ and $C \subset \alpha$. The following assertions are straightforward to check:

- $\xi(\sigma)=C$ if and only if $Q(C, \xi)$ and $Q\left(C^{\prime}, \xi\right)$ both contain $C$;

- $\xi(\sigma)=C^{\prime}$ if and only if $Q(C, \xi)$ and $Q\left(C^{\prime}, \xi\right)$ both contain $C^{\prime}$;

- $\xi(\sigma)=\sigma$ if and only if $Q(C, \xi)$ does not contain $C^{\prime}$ and vice-versa.

Now remark that $Q(y, \xi)=Q\left(y, \xi^{\prime}\right)$ for any chamber $y \in \operatorname{Ch}(A)$ in view of Lemma 2.22. Therefore, we deduce from the above that $\xi$ and $\xi^{\prime}$ coincide on $\sigma$.

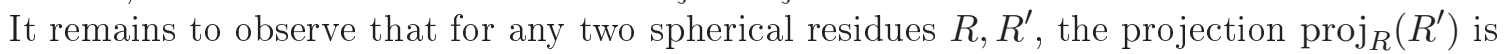
uniquely determined by the set of all projections $\operatorname{proj}_{\sigma}\left(R^{\prime}\right)$ on panels $\sigma$ containing $R$.

The next result supplements the description provided by Proposition 2.15. 
Proposition 2.27. Let $x \in \mathrm{Ch}(X), \xi \in \mathscr{C}_{\mathrm{sph}}(X)$ and $A$ be an apartment containing the sector $Q(x, \xi)$. Then we have

$$
Q(x, \xi)=\bigcap_{\alpha \in \Phi_{A}(x) \cap \Phi_{A}(\xi)} \alpha .
$$

Proof. Set $Q=Q(x, \xi)$ and $Q^{\prime}=\bigcap_{\alpha \in \Phi_{A}(x) \cap \Phi_{A}(\xi)} \alpha$. By Lemma 2.26 there is a sequence $\left(R_{n}^{\prime}\right)$ of spherical residues of $A$ converging to $\xi$ in $\mathscr{C}_{\mathrm{sph}}(A)$.

A half-apartment $\alpha$ belongs to $\Phi_{A}(\xi)$ if and only if $R_{n}^{\prime} \subset \alpha$ for any sufficiently large $n$. Therefore, the equality $Q=Q^{\prime}$ follows from Lemma 2.22.

The following result allows one to extend all the results of this section to sectors based at any spherical residues, and not only at chambers. It shows in particular that sectors based at residues which are not chamber may be thought of as sector-faces:

Corollary 2.28. Let $x \in \operatorname{Res}_{\mathrm{sph}}(X), \xi \in \mathscr{C}_{\mathrm{sph}}(X)$ and $A$ be an apartment containing the sector $Q(x, \xi)$. Then $Q(x, \xi)$ coincides with the intersection of all sectors $Q(y, \xi)$ where $y$ runs over the set of chambers of $A$ containing $x$.

Proof. By Lemma 2.26, there is a sequence $\left(R_{n}\right)$ of spherical residues of $A$ converging to $\xi$ in $\mathscr{C}_{\text {sph }}(A)$. By Proposition 2.15 we have $Q(x, \xi)=\bigcup_{k \geq 0} \bigcap_{n \geq k} \operatorname{Conv}\left(R, R_{n}\right)$.

Let $\mathcal{C}$ be the set of all chambers of $A$ containing $x$. Using the fact that the convex hull of two residues is nothing but the intersection of all roots containing them, we deduce that for any $R_{n}$ we have

$$
\operatorname{Conv}\left(x, R_{n}\right)=\bigcap_{y \in \mathcal{C}} \operatorname{Conv}\left(y, R_{n}\right) .
$$

It follows that

$$
Q(x, \xi)=\bigcup \bigcup_{k \geq 0} \bigcap_{y \in \mathcal{C}} \operatorname{Conv}\left(y, R_{n}\right) .
$$

All it remains to show is thus that

$$
\bigcup_{k \geq 0} \bigcap_{y \in \mathcal{C}} \bigcap_{n \geq k} \operatorname{Conv}\left(y, R_{n}\right)=\bigcap_{y \in \mathcal{C}} \bigcup_{k \geq 0} \bigcap_{n \geq k} \operatorname{Conv}\left(y, R_{n}\right) .
$$

To establish this equality, notice that the inclusion $\subset$ is immediate. The reverse inclusion follows similarly using the fact that $\mathcal{C}$ is a finite set.

We close this section with the following subsidiary fact.

Lemma 2.29. Let $x \in \operatorname{Res}_{\mathrm{sph}}(X), \xi \in \mathscr{C}_{\mathrm{sph}}(X)$ and $A$ be an apartment containing the sector $Q=Q(x, \xi)$. Then for all $\alpha_{1}, \ldots, \ldots \alpha_{n} \in \Phi_{A}(\xi)$, the intersection

$$
Q(x, \xi) \cap\left(\bigcap_{i=1}^{n} \alpha_{i}\right)
$$

is non-empty.

Proof. In view of Corollary 2.28, it is enough to deal with the case $x$ is a chamber. Thus we assume henceforth that $x \in \mathrm{Ch}(X)$.

If the result is true with $n=1$, then it is true for any $n$ by a straightforward induction argument using Remark 2.14. 
We need to show that the sector $Q(x, \xi)$ penetrates any $\alpha \in \Phi_{A}(\xi)$. We work by induction on the dimension of Davis' CAT(0) realisation $|X|$ of $X$ (see [Dav98]). Recall that this dimension equals the maximal possible rank of a spherical residue of $X$. We call it the dimension of $X$ for short.

Pick a sequence $\left(R_{n}\right)$ of spherical residues of $A$ converging to $\xi$ in $\mathscr{C}_{\mathrm{sph}}(A)$; such a sequence exists in view of Lemma 2.26. In order to simplify the notation, choose $\left(R_{n}\right)$ in such a way that $R_{0}=x$. Since the desired result clearly holds if $\xi$ is an interior point, namely $\xi \in \operatorname{Res}_{\operatorname{sph}}(X)$, we shall assume that $\left(R_{n}\right)$ goes to infinity.

Let $p_{n} \in\left|R_{n}\right|$. Upon extracting, the sequence $\left(p_{n}\right)$ converges to some point $\eta$ of the visual boundary $\partial_{\infty}|A| \subset \partial_{\infty}|X|$. Let $\mathcal{H}_{\eta}$ denote the set of all walls $H$ of $A$ such that $\eta \in \partial|H|$. Equivalently some geodesic ray of $|A|$ pointing to $\eta$ is contained in a tubular neighbourhood of $|H|$. Let also $W$ denote the Weyl group of $X$ (which acts on $|A|$ by isometries), and $W_{\eta}$ denote the subgroup generated by the reflections associated with the elements of $\mathcal{H}_{\eta}$. Recall from [Deo89] that $W_{\eta}$ is a Coxeter group.

Let now $\alpha \in \Phi_{A}(\xi)$. If $x \subset \alpha$, then $Q(x, \xi) \subset \alpha$ by Proposition 2.27 and we are done. We assume henceforth that $x$ is not contained in $\alpha$.

Recall that $x=R_{0}$. In particular $p_{0} \in|x|$. Therefore, Proposition 2.27 implies that the geodesic ray $\left[p_{0}, \eta\right)$ is entirely contained in $|Q(x, \xi)|$. In particular, if this ray penetrates $|\alpha|$, then we are done. We assume henceforth that this is not the case. Since $p_{n} \in|\alpha|$ for any large $n$, this implies that the wall $\partial \alpha$ belongs to $\mathcal{H}_{\eta}$.

We claim that there is some $R \in \operatorname{Res}_{\text {sph }}(A)$ such that $R \subset \alpha$ and $R \subset \beta$ for all $\beta \in$ $\Phi_{A}(x) \cap \Phi_{A}(\xi)$ such that $\partial \beta \in \mathcal{H}_{\eta}$. The proof of this claim requires to use the induction hypothesis for the thin building $A_{\eta}$ associated to the Coxeter group $W_{\eta}$. The walls and the roots of $A_{\eta}$ may be canonically and $W_{\eta}$-equivariantly identified with the elements of $\mathcal{H}_{\eta}$. This yields a well defined $W_{\eta}$-equivariant surjective map $\pi_{\eta}: \operatorname{Res}_{\mathrm{sph}}(A) \rightarrow \operatorname{Res}_{\mathrm{sph}}\left(A_{\eta}\right)$ which maps a residue $\sigma$ to the unique spherical residue which is contained in all roots $\phi$ containing $\sigma$ and such that $\partial \phi \in \mathcal{H}_{\eta}$. The way $\pi_{\eta}$ acts on $\operatorname{Ch}(A)$ is quite clear: it identifies chambers which are not separated by any wall in $\mathcal{H}_{\eta}$.

We now verify that $\operatorname{dim}\left(A_{\eta}\right)<\operatorname{dim}(A)=\operatorname{dim}(X)$. Indeed, a spherical residue in a Davis complex is minimal (i.e. does not contain properly any spherical residue) if and only if it coincides with the intersection of all walls containing it. Now, given a spherical residue of maximal possible rank $\sigma$ in $A_{\eta}$, then on the one hand the intersection in $\left|A_{\eta}\right|$ of all the walls in $\mathcal{H}_{\eta}$ containing $|\sigma|$ coincides with $|\sigma|$, but on the other hand the intersection of these same walls in $|A|$ is not compact since it contains a geodesic ray pointing to $\eta$. This shows that $\operatorname{dim}(A)>\operatorname{dim}\left(A_{\eta}\right)$ as desired.

We are now in a position to apply the induction hypothesis in $A_{\eta}$. Notice that, upon extracting, the sequence $\left(\pi_{\eta}\left(R_{n}\right)\right)$ converges in $\mathscr{C}_{\mathrm{sph}}\left(A_{\eta}\right)$ to a point which we denote by $\pi_{\eta}(\xi)$. Furthermore, we have

$$
\Phi_{A_{\eta}}\left(\pi_{\eta}(\xi)\right)=\left\{\beta \in \Phi_{A}(\xi) \mid \partial \beta \in \mathcal{H}_{\eta}\right\}
$$

By induction there is some $R^{\prime} \in \operatorname{Res}_{\mathrm{sph}}\left(A_{\eta}\right)$ contained in both $\alpha$ and $Q\left(\pi_{\eta}(x), \pi_{\eta}(\xi)\right)$. Let $R \in \operatorname{Res}_{\mathrm{sph}}(A)$ be any element such that $\pi_{\eta}(R)=R^{\prime}$. In view of Proposition 2.27, we have $R \subset \alpha$ and $R \subset \beta$ for all $\beta \in \Phi_{A}(x) \cap \Phi_{A}(\xi)$ such that $\partial \beta \in \mathcal{H}_{\eta}$, which confirms the above claim. 
Pick now $p \in|R|$ any point supported by $R$ and consider the geodesic ray $\rho$ joining $p$ to $\eta$. We shall prove that this ray penetrates $|Q(x, \xi)|$, from which the desired conclusion follows. Let $q_{n}=\rho(n)$ for all $n \geq 0$.

Suppose for a contradiction that for all $n$, we have $q_{n} \notin|Q(x, \xi)|$. Then, in view of Proposition 2.27 there exists a root $\alpha_{n} \in \Phi_{A}(x) \cap \Phi_{A}(\xi)$ which does not contain $q_{n}$.

We claim that none of the $\partial \alpha_{n}$ 's separate the ray $[x, \eta)$ from $[p, \eta)$. Indeed, if $\partial \alpha_{n}$ did, then it would belong to $\mathcal{H}_{\eta}$, which contradicts the definition of $R$.

Since $[x, \eta) \subset|Q(x, \xi)| \subset\left|\alpha_{n}\right|$ for any $n$, it follows in particular that for each $n$ there is some $n^{\prime}$ such that $q_{n^{\prime}}$ is contained in $\alpha_{n}$. Upon extracting, we may assume that either $n^{\prime}>n$ or $n^{\prime}<n$ for all $n$. In either case, it follows that the set $\left\{\alpha_{n}\right\}$ is infinite and that for any $k$, the intersection $\bigcap_{n \leq k}-\alpha_{k}$ contains some point of $q_{k}^{\prime} \in[p, \eta)$. In particular, when $k$ tends to infinity, the number of walls separating $q_{k}^{\prime}$ from $[x, \eta)$ tends to infinity, which contradicts the fact that $[x, \eta)$ and $[p, \eta)$ are at finite Hausdorff distance from one another.

2.5. Incidence properties of sectors. The goal of this section is to establish that two sectors pointing towards the same point at infinity have a non-empty intersection. This should be compared to the corresponding statement in the classical case of Euclidean buildings, see [BT72, 2.9.1].

Proposition 2.30. Let $\xi$ be any point in $\mathscr{C}_{\mathrm{sph}}(X)$. Given any two residues $x, y \in \operatorname{Res}_{\mathrm{sph}}(X)$, there exists $z \in \operatorname{Res}_{\mathrm{sph}}(X)$ such that $Q(z, \xi) \subset Q(x, \xi) \cap Q(y, \xi)$.

Proof. In view of Remark 2.14, it suffices to prove that the intersection $Q(x, \xi) \cap Q(y, \xi)$ contains some spherical residue $z$. We proceed by induction on the root-distance $d(x, y)$.

If $x \subset y$ or $y \subset x$, the result is clear. Thus there is no loss of generality in assuming that the open interval $] x, y[$ is non-empty, see Lemma 1.3. Let $z \in] x, y[$. By induction there exists $a \in Q(x, \xi) \cap Q(z, \xi)$ and $b \in Q(y, \xi) \cap Q(z, \xi)$. Therefore, it suffices to show that $Q(a, \xi) \cap Q(b, \xi)$ is non-empty. Since the sectors $Q(a, \xi)$ and $Q(b, \xi)$ are both contained in $Q(z, \xi)$, it follows in particular that they are contained in a common apartment, say $A$. Let $\alpha_{1}, \ldots, \alpha_{k}$ be the finitely many elements of $\Phi_{A}(a) \cap \Phi_{A}(\xi) \backslash \Phi_{A}(b)$. By Lemma 2.29, there is some spherical residue $R$ contained in $Q(b, \xi)$ as well as in each $\alpha_{i}$.

We claim that $R \in Q(a, \xi)$. If this were not the case, there would exist some $\alpha \in \Phi_{A}(a) \cap$ $\Phi_{A}(\xi)$ not containing $R$ in view of Proposition 2.27. The same proposition shows that if $b \subset \alpha$, then $Q(b, \xi) \subset \alpha$ which is absurd since $R \subset Q(b, \xi)$. Therefore we have $b \not \subset \alpha$ or equivalently $\alpha \notin \Phi_{A}(b)$. Thus $\alpha$ coincides with one of the $\alpha_{i}$ 's, and yields again a contradiction since $R \subset \bigcap_{i=1}^{k} \alpha_{i}$. This confirms the claim, thereby concluding the proof of the proposition.

2.6. Covering the combinatorial compactifications with apartments. We are now able to prove Proposition 2.4. In fact we shall establish the following more precise version.

Proposition 2.31. Given $\xi \in \mathscr{C}_{\mathrm{sph}}(X)$, we have the following.

(i) There exists a sequence of spherical residues $\left(x_{0}, x_{1}, \ldots\right)$ which penetrates and eventually remains in every sector pointing to $\xi$, and such that $x_{n}=\operatorname{proj}_{x_{n}}(\xi)$ for all $n$.

(ii) Every such sequence converges to $\xi$. 
Proof of Propositions 2.4 and 2.31. In view of Proposition 2.12 and the fact that a sequence as in (i) eventually remains in one apartment, it suffices to prove Proposition 2.31.

(i) Let $Q$ be some sector pointing to $\xi$ and $A$ be an apartment containing $Q$. Since $A$ is locally finite and since any finite intersection of sectors pointing to $\xi$ is non-empty by Proposition 2.30, it follows that $Q$ contains a sequence $\left(x_{n}\right)$ of spherical residues which penetrates and eventually remains in every sector contained in $A$ and pointing to $\xi$. Furthermore, upon replacing $x_{n}$ by $\operatorname{proj}_{x_{n}}(\xi)$ for all $n>0$, we may and shall assume without loss of generality that $\left(x_{n}\right)$ is eventually meets the interior of every root $\alpha \in \Phi_{A}(\xi)$ such that $-\alpha \notin \Phi_{A}(\xi)$. Notice that $\operatorname{proj}_{x_{n}}\left(\operatorname{proj}_{x_{n}}(\xi)\right)=\operatorname{proj}_{x_{n}}(\xi)$ in view of Corollary 2.19.

If $Q^{\prime}$ is any other sector pointing to $\xi$, then $Q \cap Q^{\prime}$ contains some sector by Proposition 2.30. Therefore $\left(R_{n}\right)$ eventually penetrates and remains in $Q^{\prime}$ as desired.

(ii) Let $\left(R_{n}\right)$ be a sequence of spherical residues which eventually penetrates and remains in every sector pointing to $\xi$, and such that $R_{n}=\operatorname{proj}_{R_{n}}(\xi)$ for all $n$. Then the assumption on $\left(R_{n}\right)$ ensures that the sequence $\left(R_{n}\right)$ eventually remains on one side of every wall of $A$ (see Proposition 2.27). In particular $\left(R_{n}\right)$ converges to some $\xi^{\prime} \in \mathscr{C}_{\mathrm{sph}}(A)$ by Lemma 2.3. By construction we have $\Phi_{A}(\xi) \subset \Phi_{A}\left(\xi^{\prime}\right)$. Furthermore, since the sequence $\left(R_{n}\right)$ eventually leaves every root $\alpha$ of $A$ such that $-\alpha \notin \Phi_{A}(\xi)$, we obtain in fact $\Phi_{A}(\xi)=\Phi_{A}\left(\xi^{\prime}\right)$. Thus $\xi=\xi^{\prime}$ by Lemma 2.26. Therefore we have $\operatorname{proj}_{R}(\xi)=\operatorname{proj}_{R}\left(\xi^{\prime}\right)=\operatorname{proj}_{R}\left(R_{n}\right)$ for any sufficiently large $n$.

Since $R \in \operatorname{Res}_{\mathrm{sph}}(X)$ is arbitrary, we have just established that $R_{n}$ converges to $\xi$ in $\mathscr{C}_{\mathrm{sph}}(X)$ as desired.

We have seen in Lemma 2.3 that a sequence $\left(R_{n}\right)$ contained in some apartment $A$ converges to $\xi \in \mathscr{C}_{\mathrm{sph}}(A)$ if and only if it eventually remains on one side of every wall of $A$. By Proposition 2.27, the latter is equivalent to the fact that $\left(R_{n}\right)$ eventually penetrates and remains in every sector of $A$ pointing to $\xi$. As we have just seen in the above proof, this implies that $\left(R_{n}\right)$ converges in $\mathscr{C}_{\mathrm{sph}}(X)$. Thus we have proven the following:

Corollary 2.32. Let $\left(R_{n}\right)$ be a sequence of spherical residues contained in some apartment A. If $\left(R_{n}\right)$ converges in $\mathscr{C}_{\mathrm{sph}}(A)$, then it also converges in $\mathscr{C}_{\mathrm{sph}}(X)$. In particular, it always admit a subsequence which converges in $\mathscr{C}_{\text {sph }}(X)$.

\section{Horofunction compactifications}

Let $Y$ be a proper metric space, i.e. a metric space all of whose closed balls are compact. Given a base point $y_{0} \in Y$, we define $\mathscr{F}\left(Y, y_{0}\right)$ as the space of 1 -Lipschitz maps $Y \rightarrow \mathbb{R}$ taking value 0 at $y_{0}$. The topology of pointwise convergence (which coincides with the topology of uniform convergence since $Y$ is proper $)$ turns $\mathscr{F}\left(Y, y_{0}\right)$ into a compact space. To each $p \in Y$ we attach the functions

$$
d_{p}: Y \rightarrow \mathbb{R}: y \mapsto d(p, y)
$$

and

$$
f_{p}: Y \rightarrow \mathbb{R}: y \mapsto d(p, y)-d\left(p, y_{0}\right)
$$

Then $f_{p}$ belongs to $\mathscr{F}\left(Y, y_{0}\right)$ and it is a matter of routine verifications to check that the map

$$
Y \rightarrow \mathscr{F}\left(Y, y_{0}\right): p \mapsto f_{p}
$$

is continuous and injective. We shall implicitly identify $Y$ with its image. The closure of $Y$ in $\mathscr{F}\left(Y, y_{0}\right)$ is called the horofunction compactification of $Y$. We denote it by $\mathscr{C}_{\text {horo }}(Y)$. 
Since $\mathscr{F}\left(Y, y_{0}\right)$ may be canonically identified with the quotient of the space of all 1-Lipschitz functions by the 1-dimensional subspace consisting of constant functions, it follows that the horofunction compactification is independent of the choice of the base point $y_{0}$.

It is well known that the horofunction compactification of a proper CAT(0) space coincides with the visual compactification, see [BH99, Theorem II.8.13]. In the case of a locally finite building $X$, several proper metric spaces may be viewed as realisations of $X$ : Davis' CAT $(0)$ realisation $|X|$ is one of them; the chamber graph (i.e. the set of chambers endowed with the gallery distance) is another one; the set of spherical residues $\operatorname{Res}_{\mathrm{sph}}(X)$ endowed with the root-distance is yet another. It should be expected that the respective horofunction compactifications of these metric spaces yield different spaces which may be viewed as compactifications of the building $X$. This is confirmed by the following.

Theorem 3.1. The minimal combinatorial compactification of a locally finite building $X$ is Aut $(X)$-equivariantly homeomorphic to the horofunction compactification of its chamber graph.

Similarly, the maximal combinatorial compactification of $X$ is $\operatorname{Aut}(X)$-equivariantly homeomorphic to the horofunction compactification of $\operatorname{Res}_{\mathrm{sph}}(X)$ endowed with the root-distance.

Abusing notation slightly, we shall denote by $\mathscr{C}_{\text {horo }}(X)$ the horofunction compactification of $\left(\operatorname{Res}_{\mathrm{sph}}(X), d\right)$, where $d$ denotes the root-distance. Since by definition the chamber graph embeds isometrically into $\left(\operatorname{Res}_{\mathrm{sph}}(X), d\right)$, it follows that $\mathscr{C}_{\text {horo }}(\mathrm{Ch}(X))$ is contained as a closed subset in $\mathscr{C}_{\text {horo }}(X)$. This is confirmed by combining Proposition 2.12 with Theorem 3.1.

Proof of Theorem 3.1. In one sentence, the above theorem holds because the combinatorial bordifications are defined using combinatorial projections, and the latter notion may be defined purely in terms of the root-distance (see Corollary 1.4). Here are more details.

We deal only with the maximal combinatorial compactification, the case of the minimal one being similar but easier.

Let $\left(R_{n}\right)$ and $\left(T_{n}\right)$ be two sequences of spherical residues which converge to the same point $\xi \in \mathscr{C}_{\mathrm{sph}}(X)$. We claim that the sequences $\left(f_{R_{n}}\right)$ and $\left(f_{T_{n}}\right)$ both converge in $\mathscr{C}_{\text {horo }}(X)$ and have the same limit.

Let $x \in \operatorname{Res}_{\mathrm{sph}}(X)$. We show by induction on the root-distance $d\left(x, y_{0}\right)$ between $x$ and a base point $y_{0} \operatorname{Res}_{\mathrm{sph}}(X)$ that $f_{R_{n}}(x)$ and $f_{T_{n}}(x)$ take the same value for all sufficiently large $n$. This implies the above claim.

Assume first that $x \subset y_{0}$. Let $A$ be an apartment containing $y_{0}$ and $R_{n}$. Consider the roots of $A$. Since $x \subset y_{0}$, we have

$$
\begin{aligned}
\left|\Phi_{A}\left(R_{n}, y_{0}\right)\right|-\left|\Phi_{A}\left(R_{n}, x\right)\right| & =\left|\Phi_{A}\left(R_{n}, y_{0}\right) \backslash \Phi_{A}\left(R_{n}, x\right)\right| \\
& =\left|\Phi_{A}\left(\operatorname{proj}_{x}\left(R_{n}\right), y_{0}\right)\right|,
\end{aligned}
$$

where the last equality follows since every root containing $R_{n} \cup x$ also contains $\left(\operatorname{proj}_{x}\left(R_{n}\right)\right.$, and conversely any root containing $\operatorname{proj}_{x}\left(R_{n}\right)$ but not $y_{0}$ also contains $R_{n} \cup x$. By similar arguments, one obtains

$$
\left|\Phi_{A}\left(x, R_{n}\right)\right|-\left|\Phi_{A}\left(y_{0}, R_{n}\right)\right|=\left|\Phi_{A}\left(x, \operatorname{proj}_{x}\left(R_{n}\right) \cup y_{0}\right)\right|,
$$

where $\Phi_{A}\left(x, \operatorname{proj}_{x}\left(R_{n}\right) \cup y_{0}\right)$ denotes the set of all the roots of $A$ containing $x$ but neither $\operatorname{proj}_{x}\left(R_{n}\right)$ nor $y_{0}$. Remark that the $\operatorname{projection} \operatorname{proj}_{x}\left(R_{n}\right)$ coincides with $\operatorname{proj}_{x}(\xi)$ for any sufficiently large $n$. This shows that

$$
\begin{aligned}
f_{R_{n}}(x) & =d_{R_{n}}(x)-d_{R_{n}}\left(y_{0}\right) \\
& =\frac{1}{2}\left(\left|\Phi_{A}\left(R_{n}, x\right)\right|-\left|\Phi_{A}\left(R_{n}, y_{0}\right)\right|+\left|\Phi_{A}\left(x, R_{n}\right)\right|-\left|\Phi_{A}\left(y_{0}, R_{n}\right)\right|\right)
\end{aligned}
$$


depends only on $x, y_{0}$ and $\operatorname{proj}_{x}(\xi)$ and for any large enough $n$. In particular this shows that the sequence $\left(f_{R_{n}}\right)$ converges and its limit coincides with the limit of $\left(f_{T_{n}}\right)$ as expected.

The same arguments apply to the case $x \supset y_{0}$.

Assume now that $x$ and $y_{0}$ are not contained in one another. Then the open interval $] x, y_{0}[$ is non-empty by Lemma 1.3. Let $z$ be an element of this interval. By induction the sequences

$$
n \mapsto d_{R_{n}}(z)-d_{R_{n}}\left(y_{0}\right)
$$

and

$$
n \mapsto d_{R_{n}}(x)-d_{R_{n}}(z)
$$

both converge to some value which depends only on $\xi$. Since the sum of these sequences yields $\left(f_{R_{n}}(x)\right)$, the desired result follows.

This provides a well defined $\operatorname{Aut}(X)$-equivariant map $\mathscr{C}_{\text {sph }}(X) \rightarrow \mathscr{C}_{\text {horo }}(X): \xi \mapsto f_{\xi}$. A straightforward modification of the above arguments also show that the latter map is continuous.

Let now $\left(R_{n}\right)$ be a sequence of spherical residues such that $\left(f_{R_{n}}\right)$ converges to some $f \in$ $\mathscr{C}_{\text {horo }}(X)$. Given $x \in \operatorname{Res}_{\mathrm{sph}}(X)$, the projection $\operatorname{proj}_{x}\left(R_{n}\right)$ coincides with the unique spherical residue $\sigma$ containing $x$ and such that $f_{R_{n}}(\sigma)$ is minimal with respect to the latter property (see Corollary 1.4). Since $X$ is locally finite, the set $\operatorname{St}(x)$ is finite and we deduce from the above that $\operatorname{proj}_{x}\left(R_{n}\right)$ takes a constant value, say $\xi_{f}(x)$, for all sufficiently large $n$. Furthermore, if $\left(T_{n}\right)$ were another sequence such that $\left(f_{T_{n}}\right)$ converges to $f \in \mathscr{C}_{\text {horo }}(X)$, then the same arguments shows that $\operatorname{proj}_{x}\left(T_{n}\right)$ also converges to the same $\xi_{f}(x)$. This shows that there is a well defined $A u t(X)$-equivariant map $\mathscr{C}_{\text {horo }}(X) \rightarrow \mathscr{C}_{\text {sph }}(X): f \mapsto \xi_{f}$ such that $f_{\xi_{f}}=f$ and $\xi_{f_{\xi}}=\xi$ for all $f \in \mathscr{C}_{\text {horo }}(X)$ and $\xi \in \mathscr{C}_{\text {sph }}(X)$.

Thus $\mathscr{C}_{\text {horo }}(X)$ and $\mathscr{C}_{\text {sph }}(X)$ are indeed $\operatorname{Aut}(X)$-equivariantly homeomorphic.

\section{Group-theoretic Compactifications}

4.1. The Chabauty topology. Let $G$ be a locally compact metrizable topological group and $\mathcal{S}(G)$ denote the set of closed subgroups of $G$. The reader may consult [Bou07b] for an exposition of several equivalent definitions of the Chabauty topology on $\mathcal{S}(G)$; this topology is compact (Theorem 1 of $\S 5.2$ in loc. cit), metrizable and preserved by the conjugation action of $G$. The next proposition provides a concrete way to handle convergence in this space and could be viewed as yet another definition of the Chabauty topology.

Lemma 4.1. Let $F_{n} \in \mathcal{S}(G)$ for $n \geq 1$. The sequence $\left(F_{n}\right)$ converges to $F \in \mathcal{S}(G)$ if and only if the two following conditions are satisfied:

(i) For every sequence $\left(x_{n}\right)$ such that $x_{n} \in F_{n}$, if there exists a subsequence $\left(x_{\varphi(n)}\right)$ converging to $x \in G$, then $x \in F$.

(ii) For every element $x \in F$, there exists a subsequence $\left(x_{n}\right)$ converging to $x$ and such that $x_{n} \in F_{n}$ for every $n \geq 1$.

Proof. See [GR06, Lemma 2].

4.2. Locally finite groups. Let $G$ be a topological group. The group $G$ is said topologically locally finite (or simply locally finite when there is no ambiguity) if every finitely generated subgroup of $G$ is relatively compact. Zorn's lemma allows one to define the locally finite radical of $G$ (or LF-radical), denoted $\operatorname{Rad}_{\mathrm{LF}}(G)$, as the unique subgroup of $G$, which is normal, topologically locally finite, and maximal for these properties. It may be shown that 
if $G$ is locally compact, then the closure of a locally finite subgroup is itself locally finite (see [Cap07, Lemma 2.1]). In particular, in that case the LF-radical is a closed subgroup.

One also shows that if $G$ is locally compact, then $G$ is locally finite if and only if every compact subset of $G$ topologically generates a compact subgroup of $G$ (see [Cap07, Lemma 2.3]). In particular a locally compact topologically locally finite group is amenable.

Example 4.2. Let $F$ be a non-archimedean local field, with absolute value $|\cdot|$ and ring of integers $\mathcal{O}_{F}$. In contrast with the archimedean case, the group $(F,+)$ is locally finite. Indeed, if $x_{1}, \ldots, x_{n}$ are elements of $F$, then the subgroup they generate is included in the ball centered at the origin and of radius equal to the maximum of the absolute values of the $x_{i}$.

The group $F^{\times}$is not locally finite: if $|x| \neq 1$ is different than one, then $x^{n}$ will leave every compact set as $n$ tends to $\pm \infty$. So $\operatorname{Rad}_{\mathrm{LF}}\left(F^{\times}\right) \subset \mathcal{O}_{F}^{\times}$, which is itself a compact group, and thus we have equality: $\operatorname{Rad}_{\mathrm{LF}}\left(F^{\times}\right)=\mathcal{O}_{F}^{\times}$.

Example 4.3. With the same notations as in the example above, let $P$ be the subgroup of $\mathrm{SL}_{3}(F)$ consisting of upper triangular matrices. The same argument as above proves that $\operatorname{Rad}_{\mathrm{LF}}(P)$ is included in the group $D$ of matrices of the form

$$
\left(\begin{array}{ccc}
a \in \mathcal{O}_{F}^{\times} & * \in F & * \in F \\
0 & b \in \mathcal{O}_{F}^{\times} & * \in F \\
0 & 0 & (a b)^{-1}
\end{array}\right) .
$$

It turns out that $D$ itself is locally finite. Indeed, if $A_{1}, \ldots, A_{n}$ are matrices in $D$, then a simple calculation shows that the absolute values of the elements of the upper diagonal elements in products and inverses of the $A_{i}$ are bounded. Then it follows that the upper right element is also of absolute value bounded. Hence $\operatorname{Rad}_{\mathrm{LF}}(P)=D$.

The group $D$ appears as an example of a limit group in [GR06, §6.2]. Similar calculations also prove that the other limit groups which appear in [GR06, §6.2], such as the group of matrices of the form

$$
\left(\begin{array}{ccc}
a & b & * \in F \\
c & d & * \in F \\
0 & 0 & (a d-b c)^{-1}
\end{array}\right),
$$

with $\left(\begin{array}{ll}a & b \\ c & d\end{array}\right) \in \mathrm{GL}_{2}\left(\mathcal{O}_{F}\right)$, are locally finite.

4.3. Stabilisers of points at infinity. Let $X$ be a building and $G$ be a locally compact group acting continuously by type-preserving automorphisms on $X$ in such a way that the stabiliser of every spherical residue is compact. A special case in which the latter condition automatically holds is when the $G$-action on the $\operatorname{CAT}(0)$ realisation $|X|$ is proper. In particular, this happens if $X$ is locally finite and $G$ is a closed subgroup of $\operatorname{Aut}(X)$.

The goal of this section is to provide a description of the $G$-stabilisers of points in $\mathscr{C}_{\mathrm{sph}}(X)$.

Lemma 4.4. Let $x \in \operatorname{Res}_{\mathrm{sph}}(X)$ and $\xi \in \mathscr{C}_{\mathrm{sph}}(X)$. Then any element $g \in G$ fixing $x$ and $\xi$ fixes the sector $Q(x, \xi)$ pointwise.

Proof. It is clear that $g$ stabilises $Q(x, \xi)$. Let $A$ be an apartment containing $Q(x, \xi)$ and $\rho$ be a retraction onto $A$ centred at some chamber $C$ containing $x$. Let $g_{A}: A \rightarrow A$ denote the restriction $\rho \circ g$ to $A$. Thus $g_{A}$ is a type-preserving automorphism of $A$ and all we need to show is that it fixes $Q(x, \xi)$ pointwise. Let $y \in Q(x, \xi)$. If $y \subset x$, then $y$ is fixed by $g_{A}$ since $g_{A}$ is type-preserving. If $x \subset y$, then $y$ is contained in $\operatorname{proj}_{x}(\xi)$ by Corollary 2.19 and is thus fixed by $g_{A}$. Now, in view of Lemma 1.3, the desired assertion follows from a straightforward induction on the root-distance $d(x, y)$. 
Recall that an element of a topological group is called periodic if the cyclic subgroup it generates is relatively compact.

Lemma 4.5. Let $\xi \in \mathscr{C}_{\mathrm{sph}}(X)$ and $G_{\xi}$ be its stabilizer in $G$. We have the following.

(i) The set of periodic elements $g \in G_{\xi}$ coincides with $\operatorname{Rad}_{\mathrm{LF}}\left(G_{\xi}\right)$.

(ii) For any apartment $A$ containing a sequence of spherical residues converging to $\xi$, we have

$$
\operatorname{Rad}_{\mathrm{LF}}\left(G_{\xi}\right)=\bigcup_{x \in \operatorname{Res}_{\mathrm{sph}}(X)} \operatorname{Fix}(Q(x, \xi))=\bigcup_{x \in \operatorname{Res}_{\mathrm{sph}}(A)} \operatorname{Fix}(Q(x, \xi)) .
$$

Proof. (i) Clearly, every element of $\operatorname{Rad}_{\mathrm{LF}}\left(G_{\xi}\right)$ is periodic. Conversely, let $g$ be a periodic element in $G_{\xi}$. Then $g$ fixes a point in $|X|$ by [BH99, II.2.8], and hence a spherical residue $x \in$ $\operatorname{Res}_{\mathrm{sph}}(X)$. Now, given finitely many periodic elements $g_{n}$ and denoting by $x_{n} \in \operatorname{Res}_{\mathrm{sph}}(X)$ a $g_{n}$-fixed point, the group $\left\langle g_{1}, \ldots, g_{n}\right\rangle$ fixes $\bigcap_{i=1}^{n} Q\left(x_{i}, \xi\right)$ pointwise by Lemma 4.4. In view of Proposition 2.30, the latter intersection is non-empty. Thus $\left\langle g_{1}, \ldots, g_{n}\right\rangle$ fixes a spherical residue and is thus contained in a compact subgroup of $G$. This shows in particular that the set of periodic elements forms a subgroup of $G$ which is locally finite. The desired conclusion follows.

(ii) In view of Lemma 4.4, the equality $\operatorname{Rad}_{\mathrm{LF}}\left(G_{\xi}\right)=\bigcup \operatorname{Fix}(Q(x, \xi))$ is a reformulation of (i). The inclusion $\bigcup_{x \in \operatorname{Res}_{\mathrm{sph}}(X)} \operatorname{Fix}(Q(x, \xi)) \supset \bigcup_{x \in \operatorname{Res}_{\mathrm{sph}}(A)}^{x \in \operatorname{Res} \operatorname{sph}(X)} \operatorname{Fix}(Q(x, \xi))$ is immediate and the reverse inclusion follows from Proposition 2.30.

Example 4.6. In the case of affine buildings, there are some points $\xi \in \mathscr{C}_{\text {sph }}(X)$ such that the combinatorial sectors are usual sectors. In this case, the group $G_{\xi}$ and $\operatorname{Rad}_{L F}\left(G_{\xi}\right)$ were already considered in $[\mathrm{BT} 72, \S 4]$, where they were denoted respectively $\mathfrak{B}$ and $\mathfrak{B}_{0}$.

Although we shall only need the following in the special case of sectors, it holds for arbitrary thin sub-complexes.

Lemma 4.7. Let $Y$ be a convex sub-complex of an apartment $A$ of $X$. Assume that $G$ acts strongly transitively on $X$. Then the pointwise stabiliser of $Y$ in $G$ is topologically generated by the pointwise stabilisers of those roots of $A$ which contain $Y$. Furthermore, this group acts transitively on the set of apartments containing $Y$.

Proof. As $Y$ is convex, it coincides with the intersection of roots in $A$ containing it. Let $H$ be the subgroup of $\operatorname{Fix}_{G}(Y)$ topologically generated by the pointwise stabilisers of such roots. We will first prove that $H$ is transitive on the set of apartments containing $Y$. Let $A^{\prime}$ be such an apartment.

We shall repeatedly use the following fact which is easy to verify: since the G-action is strongly transitive, given two apartments $A_{1}, A_{2}$ which share a common half-apartment $\alpha$, there is an element $g \in G$ fixing $\alpha$ pointwise and mapping $A_{1}$ to $A_{2}$.

This remark implies in particular that there is an element of $H$ which maps $A^{\prime}$ to some apartment containing a chamber of $C$ of $A$ which meets $Y$. Therefore, it suffices to prove the desired assertion for the convex hull of $C \cup Y$. In other words, we may and shall assume that $Y$ contains some chamber $C_{0}$. 
Let $C_{1}$ be a chamber of $A$ which meets $Y$ but is not contained in it. The above remark yields an element $g_{1} \in H$ which maps $A^{\prime}=: A_{0}^{\prime}$ to some apartment $A_{1}^{\prime}$ containing $C_{1}$. Proceeding inductively, one constructs sequences $\left(C_{n}\right),\left(A_{n}^{\prime}\right)$ and $\left(g_{n}\right)$ such that:

- $C_{n}$ is a chamber of $A$ not contained in $Y_{n}:=\operatorname{Conv}\left(Y \cup\left\{C_{0}, \ldots, C_{n-1}\right\}\right)$;

- $A_{n}^{\prime}$ is an apartment containing $Y_{n_{1}} \cup C_{n}$ and sharing a half-apartment with $A_{n-1}^{\prime}$;

- $g_{n}$ is an element of $H$ which maps $A_{n-1}^{\prime}$ to $A_{n}^{\prime}$.

Furthermore, these sequences are built in such a way that $A$ is covered by $\bigcup_{n} Y_{n}$. Thus for each $C^{\prime} \in \operatorname{Ch}\left(A^{\prime}\right)$ there is some large $n$ such that $\rho_{A, C}\left(C^{\prime}\right) \subset Y_{n}$ and we deduce that $h_{m}\left(C^{\prime}\right)$ is contained in $A$ for all $m>n$, where the sequence $\left(h_{m}\right)$ defined by $h_{m}=g_{m} \cdots g_{1}$. Since $H$ is compact, the sequence $\left(h_{m}\right)$ subconverges to some $h \in H$. Since the $G$-action is continuous, the above implies that $h$ maps $A^{\prime}$ to $A$, as desired.

It remains to show that $\operatorname{Fix}_{G}(Y) \subset H$. Let thus $g \in \operatorname{Fix}_{G}(Y)$ and set $A^{\prime}=g A$. There exists some $h \in H$ such that $h A^{\prime}=A$. Hence $h g A=A$ and since $h g$ fixes $Y$ pointwise, it is enough to show that the subgroup of $\operatorname{Stab}_{G}(A)$ which fixes $Y$ pointwise is contained in $H$. The latter subgroup is trivial if $Y$ contains a chamber. Otherwise it is generated by all the reflections of $\operatorname{Stab}_{G}(A)$ fixing $Y$. It is well known and easy to see how to express such a reflection as a product of three elements, which each fixes pointwise a root of $A$. Thus there reflections indeed belong to $H$, as desired.

Combining Lemmas 4.5 and 4.7, one obtains a description of the locally finite radical $\operatorname{Rad}_{L F}\left(G_{\xi}\right)$ in terms of root groups.

4.4. Description of the group-theoretic compactification. We now assume that the building $X$ thick and locally compact, i.e. of finite thickness. In particular the automorphism group $\operatorname{Aut}(X)$ of $X$, endowed with the topology of pointwise convergence, is locally compact and metrisable. Let $G<\operatorname{Aut}(X)$ be a closed subgroup consisting of type-preserving automorphisms.

We assume that $G$ acts strongly transitively on $X$, i.e. $G$ acts transitively on the set of ordered pairs $(C, A)$ where $C$ is a chamber and $A$ an apartment containing $C$. (Throughout it is implicitly understood that the only system of apartments we consider the full system.) In particular, the group $G$ is endowed with a Tits system, or $B N$-pair, see [Bro89, Ch. V]. A basic exposition of Tits systems may be found in [Bou07a, IV, 22$]$.

The group-theoretic compactification of $X$ is based on the following simple fact.

Lemma 4.8. The map $\varphi: \operatorname{Res}_{\mathrm{sph}}(X) \rightarrow \mathcal{S}(G): R \mapsto G_{R}$ which associates a residue $R$ to its stabiliser $G_{R}$ is continuous, injective, $G$-equivariant and has discrete image. In particular it is a homeomorphism onto its image.

Proof. Continuity is obvious since $\operatorname{Res}_{\mathrm{sph}}(X)$ is discrete. The fact that $\varphi$ is equivariant is equally obvious. The injectivity follows since, by strong transitivity of the action, any two distinct residues have distinct stabilisers. It only remains to show that if some sequence $\left(R_{n}\right)$ of spherical residues is not asymptotically constant, then the sequence of stabilisers $G_{R_{n}}$ does not converge to some point of the image of $\varphi$.

Let thus $\left(R_{n}\right)$ and $R$ be spherical residues such that the sequence $\left(G_{R_{n}}\right)$ converges to $G_{R}$. Suppose for a contradiction that $R_{n}$ is not eventually constant.

Assume first that $R_{n} \not \supset R$ for infinitely many $n$. Then for each such $n$ there is an element $g_{n} \in G_{R_{n}}$ which fixes a vertex of $R$ but does not fix $R$. Clearly no subsequence of $\left(g_{n}\right)$ 
may converge to any element of $G_{R}$. On the other and since each $g_{n}$ fixes a vertex of $R$, it follows that the sequence $\left(g_{n}\right)$ is relatively compact and hence sub-converges in $g$. In view of Lemma 4.1, this contradicts the fact that $\lim G_{R_{n}}=G_{R}$.

Assume now that $R_{n} \supset R$ for all but finitely many $n$ 's. Suppose for a contradiction that $R_{n} \supsetneq R$ for infinitely many $n$ 's. Since $X$ is locally finite, this implies that there is a constant subsequence $R_{\psi(n)}=R^{\prime}$ with $R^{\prime} \supsetneq R$. Now the sequence $G_{R_{\psi(n)}}$ converges to both $G_{R}$ and $G_{R^{\prime}}$, which implies the absurd equality $R=R^{\prime}$. This finishes the proof.

Definition 4.9. The closure of the image of $\varphi$ in $\mathcal{S}(G)$ is called the group-theoretic compactification of $X$. It is denoted by $\mathscr{C}_{\mathrm{gp}}(X)$.

The main result of this section is the following.

Theorem 4.10. The group-theoretic compactification $\mathscr{C}_{\mathrm{gp}}(X)$ is $\operatorname{Aut}(X)$-equivariantly homeomorphic to the maximal combinatorial compactification $\mathscr{C}_{\mathrm{sph}}(X)$. More precisely, a sequence $\left(R_{n}\right)$ of spherical residues converges to some $\xi \in \mathscr{C}_{\mathrm{sph}}(X)$ if and only if the sequence of their stabilisers $\left(G_{R_{n}}\right)$ converges to $\operatorname{Rad}_{\mathrm{LF}}\left(G_{\xi}\right)$ in the Chabauty topology.

It follows in particular that the closure of the image of the chamber-set $\operatorname{Ch}(X)$ under $\varphi$ is $\operatorname{Aut}(X)$-equivariantly homeomorphic to the minimal combinatorial compactification $\mathscr{C}_{1}(X)$ (see Proposition 2.12).

Example 4.11. The group-theoretic compactification of Bruhat-Tits buildings was already studied in [GR06]. In particular they explicitely calculate the stabilizers and limit groups. In the case of the building associated to $\mathrm{SL}_{3}$ over a local field, there is some point $\xi$ such that $G_{\xi}=P$ is the group of upper triangular matrices. The limit group is thus the group calculated in Example 4.3.

The proof of Theorem 4.10 requires some additional preparations, collected in the following intermediate results.

Lemma 4.12. Let $\left(R_{n}\right)$ be a sequence of spherical residues converging to a point $\xi \in \mathscr{C}_{\mathrm{sph}}(X)$ and such that the sequence $\left(G_{R_{n}}\right)$ converges to some closed group $D$ in $\mathscr{C}_{\mathrm{gp}}(X)$. Then $D$ fixes $\xi$.

Proof. Given $g \in D$ and $g_{n} \in G_{R_{n}}$ be a sequence which converges to $g$ (see Lemma 4.1). Let $\sigma \in \operatorname{Res}_{\mathrm{sph}}(X)$. Then we have $g_{n}^{-1} \cdot \sigma=g^{-1} . \sigma$ for $n$ large enough. Likewise, for $n$ large enough, $g \cdot\left(\xi\left(g^{-1} \sigma\right)=g_{n} \cdot\left(\xi\left(g^{-1} \sigma\right)\right)\right.$. Therefore we have $(g \cdot \xi)(\sigma)=g_{n} \xi\left(g_{n}^{-1} \sigma\right)=\left(g_{n} \cdot \xi\right)(\sigma)$ for large $n$. Now, taking $n$ so large that $\xi(\sigma)=\operatorname{proj}_{\sigma}\left(R_{n}\right)$ and $\xi\left(g^{-1} \sigma\right)=\operatorname{proj}_{g^{-1}}\left(R_{n}\right)$, we obtain sucessively

$$
\begin{aligned}
(g . \xi)(\sigma) & =g \cdot\left(\xi\left(g^{-1} \sigma\right)\right) \\
& =g_{n} \cdot\left(\xi\left(g_{n}^{-1} \sigma\right)\right) \\
& =g_{n} \cdot\left(\operatorname{proj}_{g_{n}^{-1} \sigma}\left(R_{n}\right)\right) \\
& =g_{n} \cdot\left(\operatorname{proj}_{g_{n}^{-1} \sigma}\left(g_{n}^{-1} R_{n}\right)\right) \\
& =g_{n} \cdot\left(g_{n}^{-1} \operatorname{proj}_{\sigma}\left(R_{n}\right)\right) \\
& =\operatorname{proj}_{\sigma}\left(R_{n}\right) \\
& =\xi(\sigma) .
\end{aligned}
$$

Thus $g . \xi=\xi$ as desired.

Lemma 4.13. Let $\left(R_{n}\right)$ be a sequence of spherical residues converging to $\xi \in \mathscr{C}_{\mathrm{sph}}(X)$. Then the sequence $\left(G_{R_{n}}\right)$ converges in $\mathscr{C}_{\mathrm{gp}}(X)$ and its limit coincides with $\operatorname{Rad}_{\mathrm{LF}}\left(G_{\xi}\right)$. 
Proof. Let $D$ be a cluster value of the sequence $\left(G_{R_{n}}\right)$. It suffices to prove that $D=$ $\operatorname{Rad}_{\mathrm{LF}}\left(G_{\xi}\right)$. This indeed implies that $\left(G_{R_{n}}\right)$ admits $D$ has its unique accumulation point, and hence converges to $D$.

Since $X$ is locally finite, the pointwise stabiliser of every bounded set of $X$ is open in $G$. Moreover, since $G$ acts by simplicial isometries on $|X|$, it follows that every element acts either as an elliptic or as a hyperbolic isometry. This implies that the set of elliptic isometries is closed in $G$. Notice that this set coincides with the set of periodic elements of $G{ }^{1}$ Since every element of $D$ is limit of some sequence of periodic elements by Lemma 4.1, it follows that $D$ itself is contained in the set of periodic elements. Lemmas 4.5 and 4.12 thus yield $D \subset \operatorname{Rad}_{\mathrm{LF}}\left(G_{\xi}\right)$.

In order to prove the reverse inclusion, pick $x \in X$ and let $A$ be an apartment containing $Q(x, \xi)$. By strong transitivity, there exists some $k_{n} \in G_{x}$ such that $k_{n} R_{n} \in A$. As $G_{x}<$ $G$ is compact, we may assume upon extracting that $\left(k_{n}\right)$ converges to some $k \in G_{x}$. Let $R_{n}^{\prime}=k_{n} \cdot R_{n}$. Then $\left(R_{n}^{\prime}\right)$ is contained in $A$ converges to $k . \xi$. Furthermore, $\left(G_{R_{n}^{\prime}}\right)$ converges to $k D k^{-1}$ in $\mathscr{C}_{\mathrm{gp}}(X)$.

The sequence $\left(R_{n}^{\prime}\right)$ penetrates and eventually remains in every $\alpha \in \Phi_{A}(k . \xi)$. In particular, for any sufficiently large $n$, the pointwise stabiliser $G_{(\alpha)}$ of $\alpha$ is contained in $G_{R_{n}^{\prime}}$. By Lemma 4.1, this implies that $G_{(\alpha)}<k D k^{-1}$. Conjugating by $k^{-1}$, we deduce that for all $\alpha \in \Phi_{A}(\xi)$, we have $G_{(\alpha)}<D$. In view of Lemma 4.7, this shows that $G_{(Q(x, \xi))}<D$. The desired results follows since $G_{(Q(x, \xi))}=\operatorname{Rad}_{\mathrm{LF}}\left(G_{\xi}\right)$ by Lemma 4.5.

Lemma 4.14. Let $\left(R_{n}\right)$ be a sequence of spherical residues. If the sequence $\left(G_{R_{n}}\right)$ converges to $D \in \mathscr{C}_{\mathrm{gp}}(X)$, then $\left(R_{n}\right)$ also converges in $\mathscr{C}_{\mathrm{sph}}(X)$.

Furthermore, for all $\xi, \xi^{\prime} \in \mathscr{C}_{\mathrm{sph}}(X)$, we have $\xi=\xi^{\prime}$ if and only if $\operatorname{Rad}_{\mathrm{LF}}\left(G_{\xi}\right)=\operatorname{Rad}_{\mathrm{LF}}\left(G_{\xi^{\prime}}\right)$.

Proof. Assume that $\left(G_{R_{n}}\right)$ converges. If the sequence $\left(R_{n}\right)$ has two accumulation points $\xi, \xi^{\prime} \in \mathscr{C}_{\text {sph }}(X)$, then Lemma 4.13 implies that $\operatorname{Rad}_{\mathrm{LF}}\left(G_{\xi}\right)=\operatorname{Rad}_{\mathrm{LF}}\left(G_{\xi^{\prime}}\right)$. Therefore, the Lemma will be proved if one shows that the stabilisers of two distinct points of $\mathscr{C}_{\mathrm{sph}}(X)$ have distinct LF-radicals.

Given any $\xi \in \mathscr{C}_{\mathrm{sph}}(X)$ and $x \in \operatorname{Res}_{\mathrm{sph}}(X)$, the sector $Q(x, \xi)$ coincides with the fixed-pointset of $G_{x, \xi}$ by Lemmas 4.4 and 4.7. Furthermore Lemma 4.5 implies that $G_{x, \xi}=R_{x}$, where $R=\operatorname{Rad}_{\mathrm{LF}}\left(G_{\xi}\right)$. Thus $Q(x, \xi)$ is nothing but the fixed point set of $R_{x}$ for all $x \in \operatorname{Res}_{\mathrm{sph}}(X)$. If follows that for any other $\xi^{\prime} \in \mathscr{C}_{\mathrm{sph}}(X)$ such that $\operatorname{Rad}_{\mathrm{LF}}\left(G_{\xi^{\prime}}\right)=\operatorname{Rad}_{\mathrm{LF}}\left(G_{\xi}\right)$, the respective combinatorial sectors based at any $x \in \operatorname{Res}_{\mathrm{sph}}(X)$ and associated to $\xi$ and $\xi^{\prime}$ coincide. In view of Corollary 2.19, this implies that $\xi=\xi^{\prime}$.

We are now ready for the following.

Proof of Theorem 4.10. Consider now the map

$$
\Psi: \mathscr{C}_{\mathrm{sph}}(X) \rightarrow \mathcal{S}(G): \xi \mapsto \operatorname{Rad}_{\mathrm{LF}}\left(G_{\xi}\right) .
$$

By Proposition 4.13, the map $\Psi$ takes its values in $\mathscr{C}_{\mathrm{gp}}(X)$. By Lemma 4.14, it is bijective. The $\operatorname{Aut}(X)$-equivariance is obvious. It only remains to show that $\Psi$ is continuous.

Let $\left(\xi_{n}\right)$ be a sequence of elements of $\mathscr{C}_{\mathrm{sph}}(X)$ converging to $\xi \in \mathscr{C}_{\mathrm{sph}}(X)$. We claim that every accumulation point of $\left(\Psi\left(\xi_{n}\right)\right)$ equals $\Psi(\xi)$. Let $D$ be such an accumulation point. Upon extracting, we shall assume that $\left(\Psi\left(\xi_{n}\right)\right)$ converges to $D$.

\footnotetext{
${ }^{1}$ It turns out that the latter fact is general and does not depend on the existence of an action on a CAT(0) space. Indeed, by [Wi195, Theorem 2] the set of periodic elements is closed in any totally disconnected locally compact group.
} 
Since $\xi_{n}$ belongs to $\mathscr{C}_{\mathrm{sph}}(X)$, there exist some sequences $\left(x_{m}^{n}\right)_{m}$ of spherical residues such that $\left(x_{m}^{n}\right)_{m}$ converges to $\xi_{n}$ for each $n$. A diagonal argument shows that the sequence $\left(x_{m}^{m}\right)_{m}$ converges to $\xi$. By Lemma 4.13 , we deduce that $\left(\Psi\left(x_{m}^{m}\right)\right)_{m}$ converges to $\Psi(\xi)$ while $\left(\Psi\left(x_{m}^{n}\right)\right)_{m}$ converge to $\Psi\left(\xi_{n}\right)$. Therefore, the sequence $\left(\Psi\left(x_{m}^{m}\right)\right)_{m}$ converges to $\lim _{n} \Psi\left(\xi_{n}\right)=D$. The desired equality $\Psi(\xi)=D$ follows.

\section{Comparison to the ReFined Visual BOUNDARY}

As opposed to the previous section, we do not assume here that $X$ be locally finite. In order to simplify the notation, we shall often identify $X$ with its $\mathrm{CAT}(0)$ realisation $|X|$. This will not cause any confusion. This section is devoted to the relationship between the combinatorial and visual compactifications and their variants.

5.1. Constructing buildings in horospheres. Let $\xi \in \partial_{\infty} X$ be a point in the visual boundary of $X$. In this section we present the construction of a building $X_{\xi}$ which is canonically attached to $\xi$; it is acted on by the stabiliser $G_{\xi}$ and should be viewed as a structure which is 'transverse' to the direction $\xi$. The construction goes as follows.

Let $\mathcal{A}_{\xi}$ denote the set of all apartments $A$ such that $\xi \in \partial_{\infty} A$. Let also ${ }^{\frac{1}{2}} \mathcal{A}_{\xi}$ denote the set of all half-apartments $\alpha$ such that the visual boundary of the wall $\partial \alpha$ contains $\xi$. In particular, every $\alpha \in{ }^{\frac{1}{2}} \mathcal{A}_{\xi}$ is a half-apartment of some apartment in $\mathcal{A}_{\xi}$.

Since any geodesic ray is contained in some apartment (see [CH06, Theorem E]), it follows that the set $\mathcal{A}_{\xi}$ is non-empty. This is not the case for ${ }^{\frac{1}{2}} \mathcal{A}_{\xi}$, which is in fact empty when $\xi$ is a 'generic' point at infinity. We shall not try to make this precise.

Lemma 5.1. For all $A, A^{\prime} \in \mathcal{A}_{\xi}$ and each $C \in \mathrm{Ch}(A)$ and each geodesic ray $\rho^{\prime} \subset A^{\prime}$ pointing to $\xi$, there exists an apartment $A^{\prime \prime} \in \mathcal{A}_{\xi}$ containing both $C$ and a subray of $\rho^{\prime}$.

Proof. We work by induction on $d\left(C, \operatorname{Ch}\left(A^{\prime}\right)\right)$. Let thus $C^{\prime}$ be a chamber of $A^{\prime}$ at minimal possible distance from $C$ and let $C^{\prime}=C_{0}, C_{1}, \ldots, C_{n}=C$ be a minimal gallery. The panel which separates $C_{0}$ from $C_{1}$ defines a wall in $A^{\prime}$, and there is some half-apartment $\alpha$ of $A^{\prime}$ containing a subray of $\rho^{\prime}$. Then $C_{1} \cup \alpha$ is contained in some apartment, and the desired claim follows by induction on $n$.

Given $R \in \operatorname{Res}_{\mathrm{sph}}(X)$, let $R_{\xi}$ denote the intersection of all $\alpha \in{ }^{\frac{1}{2}} \mathcal{A}_{\xi}$ such that $R \subset \alpha$. Thus, in the case of chambers, the map $C \mapsto C_{\xi}$ identifies two adjacent chambers of $X$ unless they are separated by some wall $\partial \alpha$ with $\alpha \in{ }^{\frac{1}{2}} \mathcal{A}_{\xi}$. We call two elements of $\mathcal{C}_{\xi}$ adjacent if they are the images of adjacent chambers of $X$.

Let $W$ be the Weyl group of $W$. Choose an apartment $A \in \mathcal{A}_{\xi}$ and view $W$ as a reflection group acting on $A$. The reflections associated to half-apartments $\alpha$ of $A$ which belong to $\frac{1}{2} \mathcal{A}_{\xi}$ generate a subgroup of $W$ which we denote by $W_{\xi}$. By the main result of [Deo89], the group $W_{\xi}$ is a Coxeter group and the set $\left\{C_{\xi} \mid C \in \mathrm{Ch}(A)\right\}$ endowed with the above adjacency relation is $W_{\xi}$-equivariantly isomorphic to the chamber-graph of the Coxeter complex of $W_{\xi}$.

Lemma 5.2. The Coxeter group $W_{\xi}$ depends only on $\xi$ but not on the choice of the apartment $A$.

Proof. By the above, it suffices to show that for any two $A, A^{\prime} \in \mathcal{A}_{\xi}$, the adjacency graphs of $\left\{C_{\xi} \mid C \in \mathrm{Ch}(A)\right\}$ and $\left\{C_{\xi} \mid C \in \mathrm{Ch}\left(A^{\prime}\right)\right\}$ are isomorphic. 
We claim that if the apartments $A$ and $A^{\prime}$ contain a common chamber, then the retraction $\rho$ onto $A$ based at this chamber yields such an isomorphism. Indeed $\rho$ fixes $A \cap A^{\prime}$ pointwise, and this intersection contains a ray pointing to $\xi$. This implies that for any half-apartment of $\alpha$ of $A^{\prime}$, we have $\alpha \in{ }^{\frac{1}{2}} \mathcal{A}_{\xi}$ if and only $\rho(\alpha) \in{ }^{\frac{1}{2}} \mathcal{A}_{\xi}$. This proves the claim.

In view of Lemma 5.1, the general case of arbitrary $A, A^{\prime} \in \mathcal{A}_{\xi}$ follows from the special case that has just been dealt with.

Keeping in mind the above preparation, the proof of the following result is a matter of routine verifications which are left to the reader. Lemma 5.1 ensures that two chambers of $X_{\xi}$ are contained in an apartment; this is the main axiom to check.

Proposition 5.3. The set $\mathcal{C}_{\xi}=\left\{C_{\xi} \mid C \in \mathrm{Ch}(X)\right\}$ is the chamber-set of a building of type $W_{\xi}$ which we denote by $X_{\xi}$. Its full apartment system coincides with $\mathcal{A}_{\xi}$. The map $R \mapsto R_{\xi}$ is a $G_{\xi}$-equivariant map from $\operatorname{Res}_{\mathrm{sph}}(X)$ onto $\operatorname{Res}_{\mathrm{sph}}\left(X_{\xi}\right)$ which does not increase the rootdistance.

Remark 5.4. We have $\operatorname{dim}\left(X_{\xi}\right)<\operatorname{dim}(X)$. This was established implicitly in the course of the proof of Lemma 2.29.

5.2. A stratification of the combinatorial compactifications. By Proposition 5.3 each point $\xi$ of the visual boundary of $X$ yields a building $X_{\xi}$ and it is now desirable to compare the respective combinatorial bordifications of $X$ and $X_{\xi}$.

Theorem 5.5. For each $\xi \in \partial_{\infty} X$, there is a canonical continuous injective $\operatorname{Aut}(X)_{\xi}$ equivariant map $r_{\xi}: \mathscr{C}_{\mathrm{sph}}\left(X_{\xi}\right) \rightarrow \mathscr{C}_{\mathrm{sph}}(X)$. Furthermore, identifying $\mathscr{C}_{\mathrm{sph}}\left(X_{\xi}\right)$ with its image, one has the following stratification:

$$
\mathscr{C}_{\mathrm{sph}}(X)=\operatorname{Res}_{\mathrm{sph}}(X) \cup\left(\bigcup_{\xi \in \partial_{\infty} X} \mathscr{C}_{\mathrm{sph}}\left(X_{\xi}\right)\right) .
$$

The following lemma establishes a first basic link between points at infinity in the combinatorial bordification and points in the visual boundary.

Lemma 5.6. Let $\left(R_{n}\right)$ be a sequence of spherical residues and let $\left(p_{n}\right)$ denote the sequence of their centres. Assume that $\left(R_{n}\right)$ converges to some $f \in \mathscr{C}_{\mathrm{sph}}(X)$. Then $\left(p_{n}\right)$ admits convergent subsequences. Furthermore, any accumulation point of $\left(p_{n}\right)$ lies in the visual boundary of any combinatorial sector pointing to $f$.

It is not clear a priori that $\left(p_{n}\right)$ subconverges in $X \cup \partial_{\infty} X$ since $X$ need not be locally compact.

Proof. Fix a base point $p \in X$ and let $R \in \operatorname{Res}_{\mathrm{sph}}(X)$ denote its support. For each $n$, the geodesic segment joining $p$ to $p_{n}$ is contained in $\operatorname{Conv}\left(p, p_{n}\right)$ which is geodesically convex also in the sense of $\operatorname{CAT}(0)$ geometry. Therefore, in view of Corollary 2.18, it follows that for any $r>0$ there is some $N$ such that the geodesic segment $\left[p, p_{n}\right]$ lies entirely in $Q(R, f)$ for all $n>N$. Since combinatorial sectors are contained in apartments and since apartments are locally compact, it follows that $\left(p_{n}\right)$ subconverges to some $\xi \in \partial_{\infty} X$, and the above argument implies that the geodesic ray $[p, \xi)$ is entirely contained in the sector $Q(R, f)$.

Proof of Theorem 5.5. Let $\xi \in \partial_{\infty} X, f \in \mathscr{C}_{\text {sph }}\left(X_{\xi}\right)$. We shall now define an element $\hat{f}$ : $\operatorname{Res}_{\mathrm{sph}}(X) \rightarrow \operatorname{Res}_{\mathrm{sph}}(X)$ belonging to $\prod_{\sigma \in \operatorname{Res}_{\mathrm{sph}}(X)} \operatorname{St}(\sigma)$. To this end, we proceed as follows. 
Consider the map $\operatorname{Res}_{\mathrm{sph}}(X) \rightarrow \operatorname{Res}_{\mathrm{sph}}\left(X_{\xi}\right): \sigma \mapsto \sigma_{\xi}$ which was constructed in Section 5.1. Let $\sigma \in \operatorname{Res}_{\mathrm{sph}}(X)$, let $\rho$ be a geodesic ray emanating from the centre of $\sigma$ and pointing to $\xi$ and let $A$ be an apartment containing $\rho$. Let $\Psi_{A}(\xi)$ denote the set of all half-apartments $\alpha$ of $A$ such that $\alpha \notin \mathcal{A}_{\xi}$ and $\alpha$ contains a subray of $\rho$. Notice that if $\alpha \in \Psi_{A}(\xi)$, then $-\alpha \notin \Psi_{A}(\xi)$.

Given $\tau \in \operatorname{St}\left(\sigma_{\xi}\right)$, there is a unique spherical residue $\tau^{\prime} \in \operatorname{St}(\sigma)$ such that $\left(\tau^{\prime}\right)_{\xi}=\tau$ and that $\tau^{\prime}$ is contained in every root $\alpha \in \Psi_{A}(\xi)$ containing $\sigma$. We denote this residue $\tau^{\prime}$ by $r_{\xi}(\tau)$. It is easy to see that the map $r_{\xi}: \operatorname{St}\left(\sigma_{\xi}\right) \rightarrow \operatorname{St}(\sigma)$ does not depend on the choice of the apartment $A$.

Now we define $\widehat{f} \in \prod_{\sigma \in \operatorname{Res}_{\operatorname{sph}}(X)} \operatorname{St}(\sigma)$ by

$$
\widehat{f}: \sigma \mapsto r_{\xi}\left(f\left(\sigma_{\xi}\right)\right)
$$

Notice that the definition of $\widehat{f}$ does not depend on the choice of $A$.

We claim that $\widehat{f}$ belongs to $\mathscr{C}_{\mathrm{sph}}(X)$. Indeed, let $\left(x_{n}\right)$ of spherical residues of $X_{\xi}$ converging to $f$ and contained in some apartment $A^{\prime}$ of $X_{\xi}$ (see Proposition 2.4). We may view $A^{\prime}$ as an apartment of $X$. Choose $R_{n} \in \operatorname{Res}_{\mathrm{sph}}\left(A^{\prime}\right)$ with $\left(R_{n}\right)_{\xi}=x_{n}$ in such a way that the sequence $\left(R_{n}\right)$ eventually penetrates and remains in the interior of every $\alpha \in \Psi_{A^{\prime}}(\xi)$. It is easy to see that such a sequence exists. If follows from Lemma 2.3 and Proposition 5.3 that $\left(R_{n}\right)$ converges in $\mathscr{C}_{\mathrm{sph}}\left(A^{\prime}\right)$. The fact that $\left(R_{n}\right)$ converges in $\mathscr{C}_{\mathrm{sph}}(X)$ follows from Corollary 2.32 . The fact that $\lim _{n} R_{n}$ coincides with $\widehat{f}$ follows from Lemma 2.26. This proves the claim.

We now show that $\mathscr{C}_{\text {sph }}(X)$ admits a stratification as described above. Let $h \in \mathscr{C}_{\text {sph }}(X) \backslash$ $\operatorname{Res}_{\mathrm{sph}}(X)$ and let $\left(R_{n}\right)$ be a sequence of spherical residues contained in some apartment $A$ of $X$ and converging to $h$ (see Proposition 2.4). Upon extracting, the sequence of centres of the $R_{n}$ 's converges to some $\xi \in \partial_{\infty} A$, and the sequence $\left(\left(R_{n}\right)_{\xi}\right)_{n \geq 0}$ converges in $\mathscr{C}_{\text {sph }}\left(X_{\xi}\right)$. Let $h^{\prime}$ denote its limit. Using the very definition of the map $f \mapsto \widehat{f}$, one verifies that $\widehat{h}^{\prime}=h$, which yields the desired conclusion.

5.3. Comparison to the refined visual boundary. Besides its own intrinsic CAT(0) realisation, the building $X_{\xi}$ inherits a CAT(0) realisation in a canonical way from $X$. This follows actually from a general construction which may be performed in an arbitrary CAT(0) space and which attaches a transverse $\mathrm{CAT}(0)$ space to every point in the visual boundary. This construction was described by Karpelevič in the case of symmetric spaces; it was introduced by Leeb [Lee00] in the general context of CAT(0) spaces and used recently in [Cap07] to study the structure of amenable groups acting on CAT(0) spaces. A brief description is included below.

Let $\xi \in \partial_{\infty} X$. We let $X_{\xi}^{*}$ denote the set of geodesical rays $\rho$ pointing towards $\xi$. The set $X_{\xi}^{*}$ is endowed with a pseudo-distance defined by

$$
d\left(\rho, \rho^{\prime}\right)=\inf _{t, t^{\prime} \geq 0} d\left(\rho(t), \rho^{\prime}\left(t^{\prime}\right)\right) .
$$

If $b_{\xi}$ is a Busemann function associated to $\xi$, and if the parametrisation of $\rho$ and $\rho^{\prime}$ is chosen so that $b_{\xi} \circ \rho=b_{\xi} \circ \rho^{\prime}$, then in fact $d\left(\rho, \rho^{\prime}\right)=\lim _{t \rightarrow+\infty}\left(\rho(t), \rho^{\prime}(t)\right)$. This remark justifies that $d$ is indeed a pseudo-distance.

Identifying points at distance 0 in $X_{\xi}^{*}$ yields a metric space $X_{\xi}^{\prime}$. There is no reason for this new space to be complete; its metric completion is denoted by $X_{\xi}$. There is a canonical 
projection

$$
\pi_{\xi}: X \rightarrow X_{\xi}
$$

which associates to a point $x$ the (equivalence class of the) geodesic ray from $x$ to $\xi$. It is immediate to check that $\pi_{\xi}$ is 1-Lipschitz. by

Moreover, there is a canonical morphism $\varphi_{\xi}^{\prime}: G_{\xi} \rightarrow \operatorname{Isom}\left(X_{\xi}^{\prime}\right)$, where $G=\operatorname{Isom}(X)$, defined

$$
\varphi_{\xi}^{\prime}(g) \cdot \pi_{\xi}(x)=\pi_{\xi}(g \cdot x)
$$

The space $X_{\xi}$ is CAT(0) (see [Lee00, Proposition 2.8]). Furthermore the morphism $\varphi_{\xi}$ is continuous (see [Cap07, Proposition 4.3]).

As before, the space $X_{\xi}$ is transverse to the direction $\xi$. Since each transverse space $X_{\xi}$ admits its own visual boundary, it is natural to repeat inductively the above construction and consider sequences $\left(\xi_{1}, \xi_{2}, \ldots\right)$ such that $\xi_{n+1} \in \partial_{\infty} X_{\xi_{1}, \xi_{2}, \ldots \xi_{n}}$. The next proposition shows that this inductive process terminates after finitely many steps (in the case of buildings, this should be compared to Remark 5.4):

Lemma 5.7. There exists an integer $K \in \mathbb{N}$, depending only on $X$, such that for every sequence $\left(\xi_{1}, \ldots, \xi_{K}\right)$ with $\xi_{1} \in \partial_{\infty} X$ and $\xi_{i+1} \in \partial_{\infty} X_{\xi_{1}, \ldots, \xi_{i}}$ we have $\partial_{\infty} X_{\xi_{1}, \ldots, \xi_{K}}=\varnothing$.

Proof. See the remark after [Cap07, Corollary 4.4].

The following definition is taken over from [Cap07].

Definition 5.8. The refined visual boundary of level $k$ of $X$ is the set of all sequences $\left(\xi_{1}, \ldots, \xi_{k}, x\right)$, where $\xi_{1} \in \partial_{\infty} X$ and $\xi_{i+1} \in \partial_{\infty} X_{\xi_{1}, \ldots, x_{i}}$ for all $1 \leq i \leq k-1$ and $x \in X_{\xi_{1}, \ldots, \xi_{k}}$.

The refined visual boundary of $X$ is the union over all $k \in \mathbb{N}$ of the refined boundaries of level $k$. It is denoted by $\partial_{\infty}^{\text {fine }} X$.

As mentioned earlier, in case the underlying space $X$ is a building, the transverse space $X_{\xi}$ may be viewed as a $\mathrm{CAT}(0)$ realisation of the building $X_{\xi}$ constructed combinatorially in Section 5.1. The following result shows that in some sense, the refined visual boundary is a realisation of the boundary at infinity of the combinatorial bordification.

Theorem 5.9. Let $X$ be a building. Then there is an $\operatorname{Aut}(X)$-equivariant map $\Theta: X \cup$ $\partial_{\infty}^{\text {fine }} X \rightarrow \mathscr{C}_{\text {sph }}(X)$.

Proof. There is an $\operatorname{Aut}(X)$-equivariant surjective map $\Theta: X \rightarrow \operatorname{Res}_{\mathrm{sph}}(X)$ which associates to each point its support. Recall that the support of a point $x$ may be characterised as the unique spherical residue contained in the intersection of all half-apartments containing $x$.

Let now $\xi \in \partial_{\infty} X$; we consider the space $X_{\xi}$ both as a CAT(0) space as descrived above and as a building as described in Section 5.1. By induction on $\operatorname{dim}(X)$ (see Remark 5.4) there is a well-defined $\operatorname{Aut}(X)_{\xi}$-equivariant map $\partial_{\infty}^{\text {fine }} X_{\xi} \rightarrow \mathscr{C}_{\text {sph }}\left(X_{\xi}\right)$. Upon post-composing with the map $r_{\xi}$ of Theorem 5.5, we may assume that this map takes it values in $\mathscr{C}_{\mathrm{sph}}(X)$. Since by definition, we have a partition

$$
\partial_{\infty}^{\text {fine }} X=\bigsqcup_{\xi \in \partial_{\infty} X} \partial_{\infty}^{\text {fine }} X_{\xi},
$$

the existence of the desired map $\Theta$ follows. 
Notice that it is not clear a priori (and not true in general) that this map is surjective. Indeed, it might be the case that the $\mathrm{CAT}(0)$ space $X_{\xi}$ be reduced to a single point while the associated building $X_{\xi}$ is a spherical building not reduced to a single chamber. This happens for example of $X$ is a Fuchsian building and $\xi$ is an end point of some wall.

\section{Amenability of Stabilisers}

Let $X$ be a building. The following shows the relationship between amenable subgroups of $\operatorname{Aut}(X)$ and the combinatorial bordification $\mathscr{C}_{\mathrm{sph}}(X)$.

Theorem 6.1. Let $G$ be a locally compact group acting continuously on $X$. Then some finite index subgroup of $G$ fixes a point in $\mathscr{C}_{\mathrm{sph}}(X)$.

Assume in addition that the stabiliser in $G$ of every spherical residue is compact. Then the stabiliser of any point of $\mathscr{C}_{\mathrm{sph}}(X)$ is a closed amenable subgroup.

Proof. By [Cap07, Theorem 1.4] (see also [CL08, Theorem 1.7] in case $X$ is not locally compact), the group $G$ has a finite index subgroup $G^{*}$ which fixes a point in $X \cup \partial_{\infty}^{\text {fine }} X$. Its image under the equivariant map $\Theta$ of Theorem 5.9 is thus a $G^{*}$-fixed point in the combinatorial bordification $\mathscr{C}_{\mathrm{sph}}(X)$.

Assume now that elements of $\operatorname{Res}_{\mathrm{sph}}(X)$ haves compact stabilisers in $G$ and let $f \in \mathscr{C}_{\text {sph }}(X)$. We shall prove by induction on $\operatorname{dim}(X)$ that the stabiliser $G_{f}$ fixes some point in the refined visual bordification $X \cup \partial_{\infty}^{\text {fine }} X$. The desired result on amenability will then be provided by [Cap07, Theorem 1.5] (see also the remark following Theorem 1.1 in loc. cit. as well as [CL08, Theorem 1.7] for the non-locally compact case).

If $f \in \operatorname{Res}_{\mathrm{sph}}(X)$, then $G_{f}$ fixes the centre of the residue $f$ and there is nothing to prove. Since the latter happens when $X$ is has dimension 0 , the induction can start and we assume henceforth that $f$ is a point at infinity.

Notice that combinatorial sectors are closed and convex in the CAT(0) sense. Let $\mathcal{Q}_{f}$ denote the collection of all combinatorial sectors pointing to $f$. By Proposition 2.30, the set $\mathcal{Q}_{f}$ forms a filtering family of closed convex subsets, i.e. any finite intersection of such sectors is nonempty and contains such a sector. Since $f$ lies at infinity, it follows that $\bigcap \mathcal{Q}_{f}$ is empty. It then follows from [CL08, Theorem 1.1] and [BL05, Proposition 1.4] that the intersection of the visual boundaries of all elements of $\mathcal{Q}_{f}$ admits a canonical barycentre $\xi \in \partial_{\infty} X$ which is thus fixed by $G_{f}$. In particular $G_{f}$ acts on the building $X_{\xi}$ transverse to $\xi$.

We claim that $G_{f}$ fixes a point in $\mathscr{C}_{\mathrm{sph}}\left(X_{\xi}\right)$. In order to establish it, notice first that by definition $\xi$ belongs to the visual boundary of every apartment containing a sector in $\mathcal{Q}_{f}$. Pick such an apartment $A$. Then $A$ may also be viewed as an apartment of $X_{\xi}$ and its walls in $X_{\xi}$ is a subset of its walls in $X$. By Lemmas 2.3 and 2.24 and Corollary 2.32, it follows that $f$ determines a point $f^{\prime} \in \mathscr{C}_{\text {sph }}\left(X_{\xi}\right)$. Furthermore, since $A$ contains a subsector of every element of $\mathcal{Q}_{f}$, it follows from Lemma 2.26 that $f^{\prime}$ is uniquely determined by $f$. In particular $G_{f}$ fixes $f^{\prime} \in \mathscr{C}_{\mathrm{sph}}\left(X_{\xi}\right)$ as claimed.

Since $\operatorname{dim}\left(X_{\xi}\right)<\operatorname{dim}(X)$ by Remark 5.4, it follows from the induction hypothesis that $G_{f}<G_{f^{\prime}}$ fixes a point in the refined visual bordification $X_{\xi} \cup \partial_{\infty}^{\text {fine }} X_{\xi}$. By definition, the latter embeds in the refined visual boundary $\partial_{\infty}^{\text {fine }} X$. Thus we have shown that $G_{f}$ fixes a point in the refined visual boundary of $X$ as desired. 


\section{Appendix A. Combinatorial compactifications of CAT(0) Cube complexes}

In this appendix, we outline how some of the above results may be adapted in the case of finite-dimensional CAT(0) cube complexes. Since the arguments are generally similar but easier than in the case of buildings, we do not include detailed proofs but content ourselves by referring to the appropriate arguments in the core of the text.

Let thus $X$ be such a space. The 1 -skeleton $X^{(1)}$ induces a combinatorial metric on the set of vertices $X^{(0)}$ which is usually called the $\ell^{1}$-metric. In general it does not coincide with the restriction of the CAT(0) metric. The distance between two vertices may be interpreted as the number of hyperplanes separating them.

Let $\mathcal{P}$ denote the product of all pairs $\left\{h^{+}, h^{-}\right\}$of complementary half-spaces. Then there is a canonical embedding $X^{(0)} \rightarrow \mathcal{P}$ which is defined by remembering on which side of every wall a point lies. The closure of $X^{(0)}$ in $\mathcal{P}$ is denoted by $\mathscr{C}_{\text {ultra }}(X)$. It is called the Roller compactification or ultrafilter compactification of $X$; see [Gur08, §3.3] and references therein. It is a natural analogue of the minimal combinatorial compactification of buildings introduced in the core of the paper. Notice that, as opposed to the case of buildings, the space $\mathscr{C}_{\text {ultra }}(X)$ is compact even if $X$ is not locally finite. The following result is due to U. Bader and D. Guralnick (unpublished); it should be compared to Theorem 3.1.

Proposition A.1. The ultrafilter compactification coincides with the horofunction compactification of the vertex-set $X^{(0)}$ endowed with the $\ell^{1}$ metric.

The following is an obvious adaption Lemma 2.3; it is established with the same proof.

Lemma A.2. Let $\left(v_{n}\right)$ be a sequence of vertices. Then the sequence $\left(v_{n}\right)$ in $\mathscr{C}_{\mathrm{ultra}}(X)$ if and only if for each wall $W$ there is some $N$ such that the subsequence $\left(v_{n}\right)_{n>N}$ lies entirely on one side of $W$.

This allows one to associate with every $\xi \in \mathscr{C}_{\text {ultra }}(X)$ the set $\Phi(\xi)$ of all half-spaces in which every sequence converging to $\xi$ penetrates and eventually remains in. We define the combinatorial sector based at a vertex $v$ and pointing to $\xi$ as the set

$$
Q(v, \xi)=\bigcap_{h \in \Phi(v) \cap \Phi(\xi)} h .
$$

The (combinatorial) convex hull of a set of vertices is defined as the intersection of all half-spaces containing it. Having this in mind, it is straightforward to prove that for all $v \in X^{(0)}$ and any sequence $\left(v_{n}\right)$ of vertices converging to some $\xi \in \mathscr{C}_{\text {ultra }}(X)$, we have

$$
Q(v, \xi)=\bigcup_{k \geq 0} \bigcap_{n \geq k} \operatorname{Conv}\left(v, v_{n}\right),
$$

compare Propositions 2.15 and 2.27. The key property of combinatorial sectors pointing to some $\xi \in \mathscr{C}_{\text {ultra }}(X)$ is that they form a filtering family:

Proposition A.3. Let $v, v^{\prime} \in X^{(0)}$ and $\xi \in \mathscr{C}_{\text {ultra }}(X)$. Then there exists some vertex $v^{\prime \prime}$ such that $Q\left(v^{\prime \prime}, \xi\right) \subset Q(v, \xi) \cap Q\left(v^{\prime}, \xi\right)$.

Proof. Use induction on $\operatorname{dim}(X)$ mimicking the proof of Lemma 2.29.

Assume for the moment that $X$ is locally finite; then the automorphism group $G=\operatorname{Aut}(X)$ is locally compact and we may as before consider the closure of the set of vertex-stabilisers in the Chabauty compact space $\mathcal{S}(G)$ of closed subgroups of $G$. Notice however that one 
should not expect the latter to coincide with the ultrafilter compactification in general: the most obvious reason for this is that the group-theoretic compactification need not be a genuine compactification if $G$ is to small - for example if $G$ is discrete and torsion free, the group-theoretic compactification is a singleton. In fact, as opposed to the case of buildings, where the condition of strongly transitive actions is very natural, the transitivity properties one should impose on $G$ to make sure that the group-theoretic compactification is indeed a compactification of the vertex set do not seem natural at all. Therefore we shall not pursue this here and content ourselves with the following fact.

Proposition A.4. Let $\left(v_{n}\right)$ be a sequence of vertices of $X$ converging to some $\xi \in \mathscr{C}_{\text {ultra }}(X)$. Then the sequence of stabilisers $\left(G_{v_{n}}\right)$ converges in the Chabauty topology and its limit coincides with $\operatorname{Rad}_{\mathrm{LF}}\left(G_{\xi}\right)$.

Proof. Let $D$ be an accumulation point of the sequence $\left(G_{v_{n}}\right)$. It suffices to show that $D=$ $\operatorname{Rad}_{\mathrm{LF}}\left(G_{\xi}\right)$.

The proof of Lemma 4.12 applies verbatim to the present situation and ensures that $D \subset G_{\xi}$. Moreover, by similar arguments as in Lemma 4.5, one deduces from Proposition A.3 that the set of periodic elements of $G_{\xi}$ coincides with $\operatorname{Rad}_{\mathrm{LF}}\left(G_{\xi}\right)$. Since Lemma 4.1 implies that $D$ consists of periodic elements, one obtains the inclusion $D \subset \operatorname{Rad}_{\mathrm{LF}}\left(G_{\xi}\right)$.

In order to prove the reverse inclusion, consider an element $g \in \operatorname{Rad}_{\mathrm{LF}}\left(G_{\xi}\right)$. Then $g$ is periodic and hence it fixes some cube $C$ of $X$. Since the point $\xi$ determines exactly one side of each of the walls of $C$, it follows that $g$ fixes some vertex $v$ of $C$. In particular $g$ stabilises the sector $Q(v, \xi)$. It is easy to see by induction on the distance to $v$ that $g$ fixes pointwise all vertices contained in $Q(v, \xi)$. On the other hand, Lemma A.2 implies that the sequence $\left(v_{n}\right)$ penetrates and eventually remains in $Q(v, \xi)$. Therefore, we deduce that $g$ belongs to $G_{v_{n}}$ for any sufficiently large $n$. By Lemma 4.1, this implies that $g \in D$ as desired.

We now drop off the assumption that $X$ be locally finite. The ultrafilter compactification may also be compared to the visual boundary in a similar way as in Section 5; in particular $\mathscr{C}_{\text {ultra }}(X)$ admits a stratification as in Theorem 5.5. This may be used to established the following by mimicking the proof of Theorem 6.1.

Theorem A.5. Every amenable locally compact group acting continuously on $X$ has a finite index subgroup which fixes some point in $\mathscr{C}_{\text {ultra }}(X)$.

Conversely, given a locally compact group $G$ acting continuously on $X$ in such a way that every vertex has compact stabiliser, then the stabiliser in $G$ of every point of $\mathscr{C}_{\mathrm{ultra}}(X)$ is a closed amenable subgroup.

In the special case of a discrete group $G$, this last part was established independently in $\left[\mathrm{BCG}^{+}\right]$. Remark that, as in the case of buildings, a closed subgroup $H<G$ is amenable if and only if $H / \operatorname{Rad}_{\mathrm{LF}}(H)$ is virtually Abelian (see [Cap07], as well as [CL08, Theorem 1.7] for the non-locally compact case)

\section{REFERENCES}

[AB08] Peter Abramenko and Kenneth S. Brown. Buildings, volume 248 of Graduate Texts in Mathematics. Springer, New York, 2008. Theory and applications. 5, 7

$\left[\mathrm{BCG}^{+}\right]$J. Brodzki, S.J. Campbell, E. Guentner, G. Niblo, and N.J. Wright. Property A and CAT(0) cube complexes. Journal of Functional Analysis (to appear). 34

[BGS85] Werner Ballmann, Mikhaïl Gromov, and Viktor Schroeder. Manifolds of nonpositive curvature, volume 61 of Progress in Mathematics. Birkhäuser Boston Inc., Boston, MA, 1985. 2 
[BH99] Martin R. Bridson and André Haefliger. Metric spaces of non-positive curvature, volume 319 of Grundlehren der Mathematischen Wissenschaften. Springer-Verlag, Berlin, 1999. 2, 21, 24

[BJ06] Armand Borel and Lizhen Ji. Compactifications of symmetric and locally symmetric spaces. Mathematics: Theory \& Applications. Birkhäuser Boston Inc., Boston, MA, 2006. 3

[BL05] Andreas Balser and Alexander Lytchak. Centers of convex subsets of buildings. Ann. Global Anal. Geom., 28(2):201-209, 2005. 32

[Bou07a] Nicolas Bourbaki. Groupes et Algèbres de Lie, Chapitre IV-VI. Springer-Verlag, 2007. 25

[Bou07b] Nicolas Bourbaki. Intégration, Chapitre VIII. Springer-Verlag, 2007. 22

[Bro89] Kenneth S. Brown. Buildings. Springer-Verlag, New York, 1989. 11, 25

[BT72] F. Bruhat and J. Tits. Groupes réductifs sur un corps local. Inst. Hautes Études Sci. Publ. Math., 41:5-251, 1972. 19, 24

[Cap07] Pierre-Emmanuel Caprace. Amenable groups and Hadamard spaces with a totally disconnected isometry group. Comment. Math. Helv, To appear, 2007. 3, 4, 23, 30, 31, 32, 34

[CH06] Pierre-Emmanuel Caprace and Frederic Haglund. On geometric flats in the CAT(0) realization of Coxeter groups and Tits buildings. Canadian J. Math., Preprint, 2006. 28

[CL08] Pierre-Emmanuel Caprace and Alexander Lytchak. At infinity of finite-dimensional CAT(0) spaces. Preprint, 2008. 32, 34

[Dav98] Michael W. Davis. Buildings are CAT(0). In Geometry and cohomology in group theory (Durham, 1994), volume 252 of London Math. Soc. Lecture Note Ser., pages 108-123. Cambridge Univ. Press, Cambridge, 1998. 6, 18

[Deo89] Vinay V. Deodhar. A note on subgroups generated by reflections in Coxeter groups. Arch. Math. (Basel), 53(6):543-546, 1989. 18, 28

[GdlH90] Étienne Ghys and Pierre de la Harpe. L'action au bord des isométries. In Sur les groupes hyperboliques d'après Mikhael Gromov (Bern, 1988), volume 83 of Progr. Math., pages 135-163. Birkhäuser Boston, Boston, MA, 1990. 10

[GJT98] Yves Guivarc'h, Lizhen Ji, and J. C. Taylor. Compactifications of symmetric spaces, volume 156 of Progress in Mathematics. Birkhäuser Boston Inc., Boston, MA, 1998. 3

[GR06] Yves Guivarc'h and Bertrand Rémy. Group-theoretic compactification of Bruhat-Tits buildings. Annales scientifiques de l'ENS, 39:871-920, 2006. 1, 3, 10, 22, 23, 26

[Gur08] Dan Guralnick. Coarse decompositions for boundaries of CAT(0) groups. Preprint, 2008. 33

[Kat92] Svetlana Katok. Fuchsian groups. Chicago Lectures in Mathematics. University of Chicago Press, Chicago, IL, 1992. 10

[Lan96] Erasmus Landvogt. A compactification of the Bruhat-Tits building, volume 1619 of Lecture Notes in Mathematics. Springer-Verlag, Berlin, 1996. 3

[Lee00] Bernhard Leeb. A characterization of irreducible symmetric spaces and Euclidean buildings of higher rank by their asymptotic geometry. Bonner Mathematische Schriften [Bonn Mathematical Publications], 326. Universität Bonn Mathematisches Institut, Bonn, 2000. 30, 31

[Ron89] Mark Ronan. Lectures on buildings, volume 7 of Perspectives in Mathematics. Academic Press Inc., Boston, MA, 1989. 11, 12

[Tit74] Jacques Tits. Buildings of spherical type and finite BN-pairs. Lecture Notes in Mathematics, Vol. 386. Springer-Verlag, Berlin, 1974. 4, 5

[Tit81] J. Tits. A local approach to buildings. In The geometric vein, pages 519-547. Springer, New York, 1981. 12

[Wi195] George Willis. Totally disconnected groups and proofs of conjectures of Hofmann and Mukherjea. Bull. Austral. Math. Soc., 51(3):489-494, 1995. 27

Université Catholique de Louvain, Département de Mathématiques, Chemin du Cyclotron 2, 1348 Louvain-La-Neuve, Belgium

E-mail address: pe.caprace@uclouvain.be

Université de Lyon; Université Lyon 1; inSA de Lyon; Ecole Centrale de lyon; CNRS, UMR5208, Institut Camille Jordan, 43 Blvd du 11 novembre 1918, F-69622 Villeurbanne-Cedex, FRANCE

E-mail address: lecureux@math.univ-lyon1.fr 